\title{
ANÁLISIS ACÚSTICO DE [řr] EN ALGUNAS ZONAS DE HISPANOAMÉRICA
}

El objeto de nuestro trabajo es el de caracterizar acústicamente la realización asibilada del fonema $|\bar{r}|$. Descripciones articulatorias pueden verse en los trabajos de Amado Alonso, De geografia fonética ${ }^{1}$, y de Navarro Tomás ${ }^{2}$. El área geográfica en América de este fenómeno fue señalada por Daniel N. Cárdenas en su artículo The Geographic Distribulion of the Assibilaled R. RR in Spanish America ${ }^{3}$, basado en la bibliografía existente hasta el momento. Iamentablemente faltan aún hoy muchos datos para perfilar su distribución geográfica, social, e incluso lingüística.

Nuestras investigaciones no se han realizado in situ: hemos aprovechado la corta estancia en Madrid de algunas personas de Hispanoamérica para realizar las grabaciones. Hemos operado sobre cuatro informantes cultos, profesores todos en sus respectivos países. Su procedencia geográfica es la siguiente: de Argentina, dos: ROM, varón, nacido en Eil Chaco, y RGM, mujer, natural de San Juan. Uno de Costa Rica: OD, mujer, natural de San José. Otro de Chile: MOM, mujer, natural de Coquimbo, donde vivió hasta los diez años; desde entonces vive en Antofagasta. La edad de todos los informantes oscila entre los 30 y los 35 años.

Las grabaciones se han realizado en el Laboratorio de Fonética de Madrid. Los informantes han leido una serie de palabras en las que el

1 Rn su libro Estudios ling ̈̈isticos. Temas hispanoamericanos. Madrid, Gredos, I96r, pp. I23-r 58 .

- Manual de promunciación española. MIadrid, C. S. I. C., 1970, § II7.

- Orbis, I958, VII, pp. 407-4I4. Contiene toda la bibliografia sobre el particular. Sólo hay que añadir el libro de Dríos L. CANYIELD, La pronunciación del español en América, Bogotá, 1963, y el del mismo CínDENas, El español de Jalisco, Madrid, C. S. I. C., r967.

Para las causas fisiológicas que pueden motivar el cambio $[\overline{\mathrm{r}}]>[\check{\mathrm{r}}]$, véase el artículo de Georges Straka L'hislorie de la consonne $r$ en frangais. Neuphilologische Mitteilungen, 1965, pp. 572-606. 
fonema-problema se encontraba en todos los contornos permisibles en español. Estas palabras se hallaban situadas en 1 a $\mathrm{misma}$ si tua ción dentro de idéntica frase, con el objeto de que su posición rítmico-tonal fuese constante ${ }^{2}$. El ritmo y el modo de elocución de los informantes fue sicmpre normal.

Los análisis acústicos se han realizado con el Sona-Graph 606 IB de la Kay Electric Co. Del sonograma hemos obtenido los valores de los siguientes índices:

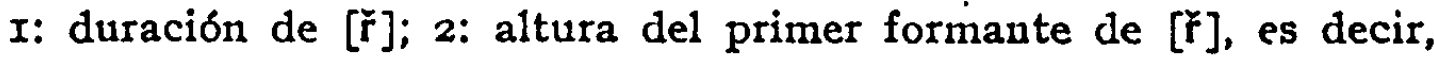
límite superior del formante; expresado en $\mathrm{Hz}$; 3: valor en $\mathrm{Hz}$ del segundo formante de $\left[\check{r}\right.$ ]; 4: naturaleza armónica o inarmónica de este $F_{2}$; .5: comienzo, medido en $\mathrm{Hz}$, de la turbulencia propia de la fricación; 6: valores en $\mathrm{Hz}$ de los dos primeros formantes de las vocales adyacentes; 7: grado positivo o negativo de las transiciones de los dos primeros formantes de las vocales adyacentes.

Las posiciones de [r] estudiadas han sido las siguientes: $\mathrm{I}$ : inicial, tras pausa; 2: en posición intervocálica; 3: en posición inicial de palabra sin pausa precedente; 4: en interior de palabra, tras $\mid s /$; 5: en interior de palabra, tras $|m| ; 6$ : en interior de palabra, tras $|l|$. Son los casos en que en español se produce la realización [ĩ]. En ninguno de los informantes se produjo asibilación del fonema $/ r /$. Sí se ha asibilado en algún caso la vibrante en posición postnuclear ante pausa.

I. [r̆-] en posición inicial, precedido de pausa.

En posición inicial, después de pausa ${ }^{2}$, hemos obtenido los siguientes valores para $[\check{r}]$ :

r. [ríma]

\begin{tabular}{|c|c|c|c|c|c|c|c|c|c|}
\hline \multirow{2}{*}{ Inf. } & \multirow{2}{*}{ r } & \multirow{2}{*}{2} & \multirow{2}{*}{3} & \multirow{2}{*}{4} & \multirow{2}{*}{5} & \multicolumn{2}{|c|}{6} & \multicolumn{2}{|c|}{7} \\
\hline & & & & & & $F_{1}$ & $F_{2}$ & $T_{1}$ & $T_{2}$ \\
\hline ROM & II,5 & 486 & I. 498,5 & arm. & I. 782 & 243 & $\mathrm{r} .822,5$ & - & - \\
\hline RGM & $I X, 5$ & - & 2.430 & inar. & 2.835 & 243 & 2.997 & - & - \\
\hline OD & 7 & $5^{67}$ & I.620 & and. & $2.146,5$ & 769 & 2.349 & - & - \\
\hline MOM & $I_{4}$ & 526, & $1.660,5$ & inar. & 2.227 .5 & 405 & $2.308,5$ & - & - \\
\hline
\end{tabular}

1 V. ILSE LEIISTE, Acouslical Characteristics of Selected English Consonants. Arouton, I964, p. I.

- Las frases que se han utilizado son: trizaba el pelo", "rima bien", trema con. 


\section{2. [risába]}

\begin{tabular}{|c|c|c|c|c|c|c|c|c|c|}
\hline \multirow{2}{*}{ Inf. } & \multirow{2}{*}{$x$} & \multirow{2}{*}{2} & \multirow{2}{*}{3} & \multirow{2}{*}{4} & \multirow{2}{*}{5} & \multicolumn{2}{|c|}{6} & \multicolumn{2}{|c|}{7} \\
\hline & & & & & & $i_{1}$ & $I_{2}$ & $r_{1}$ & $\mathrm{~T}$ \\
\hline ROM & 8,4 & 526 & $1.336,5$ & arm. & .I.70I & 324 & r. 984 & - & - \\
\hline $\mathrm{RGM}$ & $r_{4}$ & 729 & 1.944 & arm. & 2.592 & 243 & 2.754 & - & - \\
\hline OD & 4.5 & - & - & - & 1.296 & 324 & I. 782 & - & - \\
\hline MOM & 9, I & 567 & I.701 & arm. & 2.146 & 364,5 & 2.187 & - & - \\
\hline
\end{tabular}

3. [réma]

\begin{tabular}{|c|c|c|c|c|c|c|c|c|c|}
\hline \multirow{2}{*}{ Inf. } & \multirow{2}{*}{ I } & \multirow{2}{*}{2} & \multirow{2}{*}{3} & \multirow{2}{*}{4} & \multirow{2}{*}{5} & \multicolumn{2}{|c|}{6} & \multicolumn{2}{|c|}{7} \\
\hline & & & & & & $I_{1}$ & $I_{2}$ & $T_{1}$ & $\mathrm{l}_{2}$ \\
\hline ROM & 10 & 445,5 & 1.539 & arm. & $\mathrm{I} .74^{1}, 5$ & 324 & I. 782 & - & 一 \\
\hline $\mathrm{RGM}$ & 14.7 & 729 & 2.268 & arm. & 2.754 & 445.5 & 2.673 & - & - \\
\hline $\mathrm{OD}$ & 8 & 526.5 & I. $4 \times 7$ & inar. & $2.146,5$ & 405 & 2.146 & 一 & - \\
\hline MOM & $\mathrm{I} 2$ & $5^{26,5}$ & $x .660,5$ & arm. & 2.592 & $36_{4}$ & I. 863 & - & - \\
\hline
\end{tabular}

\section{4. [resciba]}

\begin{tabular}{|c|c|c|c|c|c|c|c|c|c|}
\hline \multirow{2}{*}{ Inf. } & \multirow{2}{*}{ I } & \multirow{2}{*}{2} & \multirow[b]{2}{*}{3} & \multirow[b]{2}{*}{4} & \multirow[b]{2}{*}{5} & \multicolumn{2}{|c|}{6} & \multicolumn{2}{|c|}{7} \\
\hline & & & & & & $F_{1}$ & $\mathrm{I}_{2}$ & $T_{1}$ & $\mathrm{~T}_{8}$ \\
\hline ROM & 7 & - & $x .377$ & inar. & 1. 620 & 324 & $x .741,5$ & - & 一 \\
\hline RGM & 10,5 & 567 & 2.187 & inar. & 2.835 & 405 & 2.673 & - & - \\
\hline OD & 2,8 & 688 & I. 539 & arm. & 2.835 & 445.5 & 1.620 & - & - \\
\hline MOM & 8,5 & 526,5 & I. $70 \mathrm{r}$ & arm. & 2.065 & 405 & I. 782 & - & - \\
\hline
\end{tabular}

los remos", "rezaba el rosario", "riscate bien», "rajaba con la navaja", "Roma es la capital de Italia", "rozar con el remo", "rubor de doncella", "ruso de Rusian. Se.ha analizado la vibrante de las palabras en itálica, que se presenta tanto en silaba tónica como átona. 
5. [riskale $]\left({ }^{1}\right)$

\begin{tabular}{|c|c|c|c|c|c|c|c|c|c|}
\hline \multirow{2}{*}{ Inf. } & \multirow{2}{*}{ I } & \multirow{2}{*}{2} & \multirow{2}{*}{3} & \multirow{2}{*}{4} & \multirow{2}{*}{5} & \multicolumn{2}{|c|}{6} & \multicolumn{2}{|c|}{7} \\
\hline & & & & & & $F_{1}$ & $I_{3}$ & $T_{1}$ & $T_{2}$ \\
\hline ROM & 8.5 & 480 & 1. 822,5 & arw. & 2.268 & 283.5 & $\mathrm{r} .215$ & 一 & + \\
\hline RGM & $I x$ & 405 & - & - & 3.400 & $89 x$ & I. 539 & - & + \\
\hline$O D$ & 4,2 & 729 & $I .498,5$ & inar. & $2.55 \pi .5$ & 931 & 1.782 & - & - \\
\hline MOM & 8,5 & 405 & - & - & 2.025 & $89 x$ & I. 660,5 & - & $=$ \\
\hline
\end{tabular}

6. [raxdba]

\begin{tabular}{|c|c|c|c|c|c|c|c|c|c|}
\hline \multirow{2}{*}{ Inf. } & \multirow{2}{*}{ I } & \multirow{2}{*}{2} & \multirow{2}{*}{3} & \multirow{2}{*}{4} & \multirow{2}{*}{5} & \multicolumn{2}{|c|}{6} & \multicolumn{2}{|c|}{7} \\
\hline & & & & & & $F_{x}$ & $F_{2}$ & $T_{1}$ & $x_{2}$ \\
\hline ROAI & 9 & - & $\mathrm{I} .86_{3}$ & anu. & 2.227 .5 & 283,5 & $x .2 I 5$ & - & + \\
\hline RGM & 10,5 & - & 2.187 & arm. & 2.835 & 972 & $x .782$ & - & + \\
\hline OD & 2,8 & - & - & - & $2.308,5$ & I.053 & $x .620$ & - & + \\
\hline MOAI & 9 & 567 & 1.620 & arm. & 2.146 .5 & 850 & $1.74 \pi, 5$ & - & - \\
\hline
\end{tabular}

7. [róma $]$

\begin{tabular}{|c|c|c|c|c|c|c|c|c|c|}
\hline \multirow{2}{*}{ Inf. } & \multirow{2}{*}{ I } & \multirow{2}{*}{2} & \multirow{2}{*}{3} & \multirow{2}{*}{4} & \multirow{2}{*}{5} & \multicolumn{2}{|c|}{6} & \multicolumn{2}{|c|}{7} \\
\hline & & & & & & $F_{1}$ & $\mathrm{~F}_{2}$ & $\mathrm{~T}_{1}$ & $\mathrm{~T}_{\mathbf{2}}$ \\
\hline ROAI & 10,5 & 486 & I.I 74.5 & arm. & I. $45^{8}$ & 405 & $1.012,5$ & - & + \\
\hline RGM & 15.5 & 445 & 2.025 & inar. & 2.592 & 567 & 1.053 & 一 & + \\
\hline OD & 7.7 & 526,5 & I. 336.5 & inar. & $2.146,5$ & 526 & I.255,5 & - & + \\
\hline MOMI & ro & 567 & I. $33^{6,5}$ & arm. & $x \cdot 74 \mathrm{r}, 5$ & 486 & I.2 I5 & - & + \\
\hline
\end{tabular}

1 El iuf. ROM ha realizado [raskáte] a causa del voseo . 
8. [rosdr]

\begin{tabular}{|c|c|c|c|c|c|c|c|c|c|}
\hline \multirow{2}{*}{ Iuf. } & \multirow{2}{*}{ I } & \multirow{2}{*}{2} & \multirow{2}{*}{3} & \multirow{2}{*}{4} & \multirow{2}{*}{5} & \multicolumn{2}{|c|}{6} & \multicolumn{2}{|c|}{7} \\
\hline & & & & & & $l_{1}$ & $I_{2}$ & $\mathrm{I}_{1}$ & $\mathrm{~T}_{2}$ \\
\hline ROM & 7 & $4^{86}$ & I.053 & arm. & I.296 & 202,5 & I. 34 & 一 & + \\
\hline $\mathrm{RGM}$ & 9,8 & 567 & 2.349 & inar. & 2.673 & 567 & $1.012,5$ & - & + \\
\hline OD & 6,3 & - & - & - & I. 74 I & 567 & I. 774.5 & - & + \\
\hline MOM & 5,2 & - & - & 一 & $\mathrm{I}, 822,5$ & 405 & $x .093 .5$ & - & + \\
\hline
\end{tabular}

9. [riso]

\begin{tabular}{|c|c|c|c|c|c|c|c|c|c|}
\hline \multirow{2}{*}{ Inf. } & \multirow{2}{*}{$I$} & \multirow{2}{*}{2} & \multirow{2}{*}{3} & \multirow{2}{*}{4} & \multirow[b]{2}{*}{5} & \multicolumn{2}{|c|}{6} & \multicolumn{2}{|c|}{7} \\
\hline & & & & & & $\mathrm{I}_{1}$ & $\mathrm{I}_{2}$ & $\mathrm{~T}_{1}$ & I', \\
\hline ROM & 9,8 & $4^{86}$ & I.255.5 & arm. & $1.498,5$ & 243 & 1.053 & - & + \\
\hline RGAI & 12 & 729 & 1.620 & inar. & 2.025 & 324 & 1.053 & - & + \\
\hline OD & 10,2 & $5^{67}$ & I. 377 & inar. & I. 782 & 364 & 850 & - & + \\
\hline MOM & 7.7 & $89 r$ & I. 336,5 & arm. & I. 579.5 & 405 & I.053 & - & - \\
\hline
\end{tabular}

Io. [rutuór $]$

\begin{tabular}{|c|c|c|c|c|c|c|c|c|c|}
\hline \multirow{2}{*}{ Inf. } & \multirow{2}{*}{ I } & \multirow{2}{*}{2} & \multirow{2}{*}{3} & \multirow{2}{*}{4} & \multirow{2}{*}{5} & \multicolumn{2}{|c|}{6} & \multicolumn{2}{|c|}{7} \\
\hline & & & & & & $F_{1}$ & $\mathrm{~F}_{2}$ & $\mathrm{I}_{1}$ & $\mathrm{~T}_{2}$ \\
\hline ROM & 9 & 486 & 1.215 & arm. & $\mathrm{I} .498,5$ & 283.5 & 810 & - & + \\
\hline RGM & 9 & - & - & - & 3.807 & 364.5 & 972 & - & + \\
\hline OD & 10,8 & $4^{86}$ & I. 336 & inar. & I.944 & 324 & 972 & - & + \\
\hline MOM & 7.7 & 567 & I. 4 I 7,5 & arm. & r. $70 \mathrm{r}$ & 364.5 & I.053 & - & + \\
\hline
\end{tabular}

CONCLUSIONIS

De los cuadros expuestos, podemos deducir las siguientes conclusiones:

I. La duración de $\left[\breve{r}_{-}\right]$en sílaba tónica es mayor que en sílaba átona (característica cuantitativa general, que era de prever). En sílaba tónica, la duración media es de: I2,8 c. s.; en sílaba átona, 8 c. s. 
2. De los 40 casos analizados en este grupo, nueve han resultado [r]; el resto son sonoros. En los casos sonoros, la anchura del $F_{1}$ es considerable, propiedad que, como se sabe, caracteriza a las consonantes lifuidas. Il lifuite superior de este $F_{1}$, alcanza una media de: $557 \mathrm{~Hz}$.

3. En la rcalización vibrante múltiple [ī], aparece un $\mathrm{F}_{2}$ en cada uno de los breves elementos vocálicos situados entre las oclusiones cuya frecuencia varía conforme a su contorno vocálico: con /i/ aparece a los I.644.3 Hz; con /e/ a unos. I.549 Hz; con /a/ a I.500 Hz; con /o/ a $1.247 \mathrm{~Hz}$, y con $/ \mathrm{u} / \mathrm{a}$ unos I.200 Hz. Todos ellos son valores medios.

La diferencia del $F_{2}$ entre $[\bar{r}]$ y [r] reside en que en el primer caso está interrumpido por las oclusiones, mientras que en el segundo, al ser fricativa, es continuo.

De los 40 casos analizados en este apartado sobre $[\check{r}-], F_{2}$ no aparece en siete.

Los valores medios de estos segundos formantes de [r̆ $]$ son los siguicntes: con $/ \mathrm{i} /: \mathrm{I} .74 \mathrm{I}, 5 \mathrm{~Hz}$; con $/ \mathrm{e} /: \mathrm{I.7II}, 5 \mathrm{~Hz}$; con $/ \mathrm{a} /: 1.798 \mathrm{~Hz}$; con /o/: I. $548 \mathrm{~Hz} ; \operatorname{con} / \mathrm{u} /: \mathrm{I} .366 \mathrm{~Hz}$.

No existe un paralelismo total entre los valores frecuenciales del $F_{2}$ en [î] y [ř ], pero sf lo hay en cuanto a su posición. En ambos alófonos líquidos, $\mathrm{F}_{2}$ desciende a medida que lo hace el análogo de la vocal siguiente (excepto con $/ \mathrm{a} /$ ).

4. Este $\mathrm{F}_{2}$ aparece a veces con una conformación claramente armónica; otras, no. Cuando es inarmónico, se asemeja a una concentración de energía como la que aparece en algunas realizaciones de $/ \mathrm{s} /$. (De este problema trataremos más adelante.) En $2 I$ casos $F_{2}$ es armónico; en 12 , inarmónico; en 7 , no ha aparecido.

Cuando $F_{2}$ de $[\check{r}]$ es armónico, su frecuencia es más ḅaja (valor medio, $1.575,6 \mathrm{I} \mathrm{Hz}$ ) que cuando es inarmónico (valor medio, I.975,29 Hz); asimismo, cuando es armónico, el comienzo de la fricación de [ř ] es más bajo (valor medio, 2.028,8 Hz) que cuando aparece como inarmónico (valor medio, $2.656,12 \mathrm{~Hz}$ ); cuando $\mathrm{F}_{2}$ de $[\check{r}]$ no aparece, el valor del comieuzo de la fricación es de $2.342,85 \mathrm{~Hz}$.

5. El comienzo de la fricación se produce a las siguientes frecuencias: con /i/: 2.090,6 Hz; con /e/: $2.323,5 \mathrm{~Hz}$; con /a/: $2.470 \mathrm{~Hz}$; con /o/: I.933.7 Hz; con /u/: $2.294 \mathrm{~Hz}$.

6. La transición del $\mathrm{F}_{2}$ de las vocales adyacentes muestra los siguientes movimientos:

El $F_{2}$ de /i/ está siempre por encima del $F_{2}$ de $[\check{r}]$, y $T_{2}$ es (-). Lo mismo ocurre con $/ \mathrm{e} /$. 


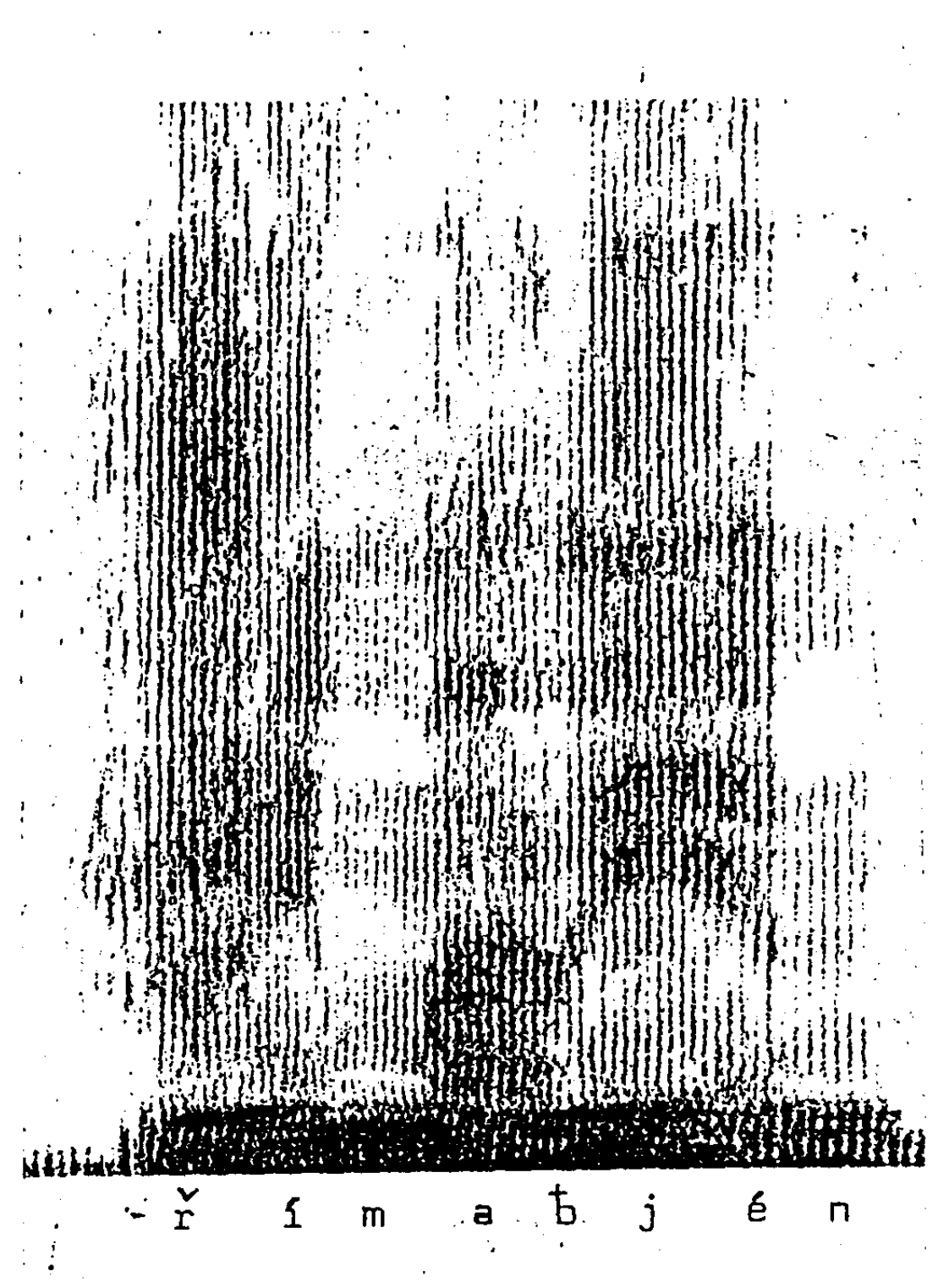

INI:. I. I.

Informante ROM. [rimabjén] Rima bien. Realización asibilada, plenamente sonora. Iil $\mathrm{F}_{\text {, }}$ cle ji llega casi a la altura del $F$, de $[i$ ! 


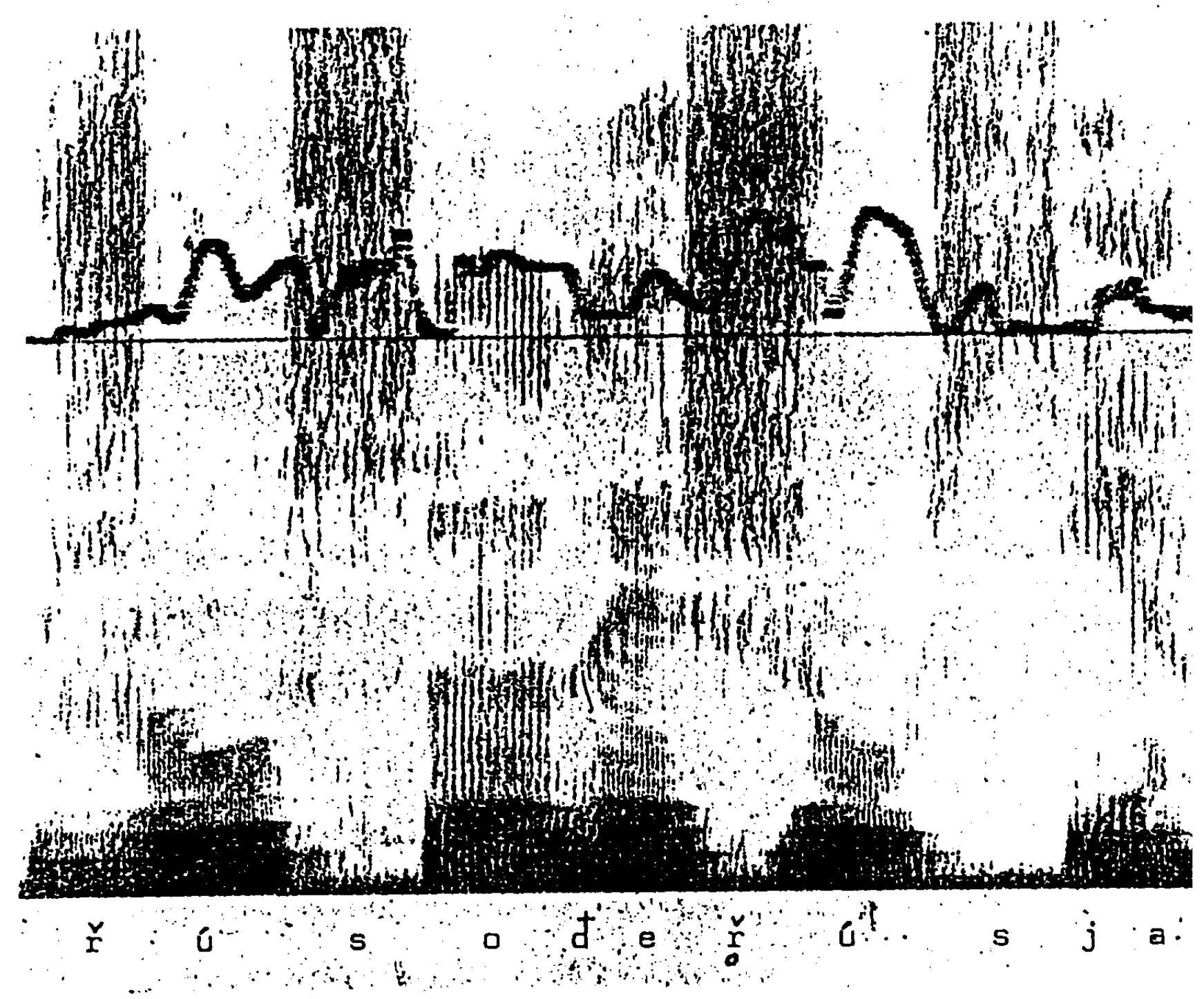

lisi. I.2.

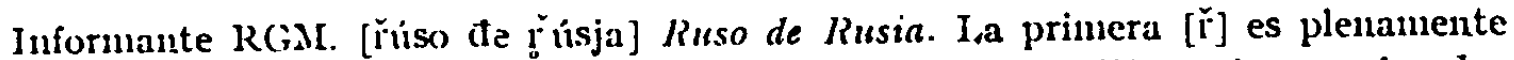
sonora: la segunda está alyo ensordecida. Obsérvense las diferencias con las clos realizaciones de $/ \mathbf{s}$. 

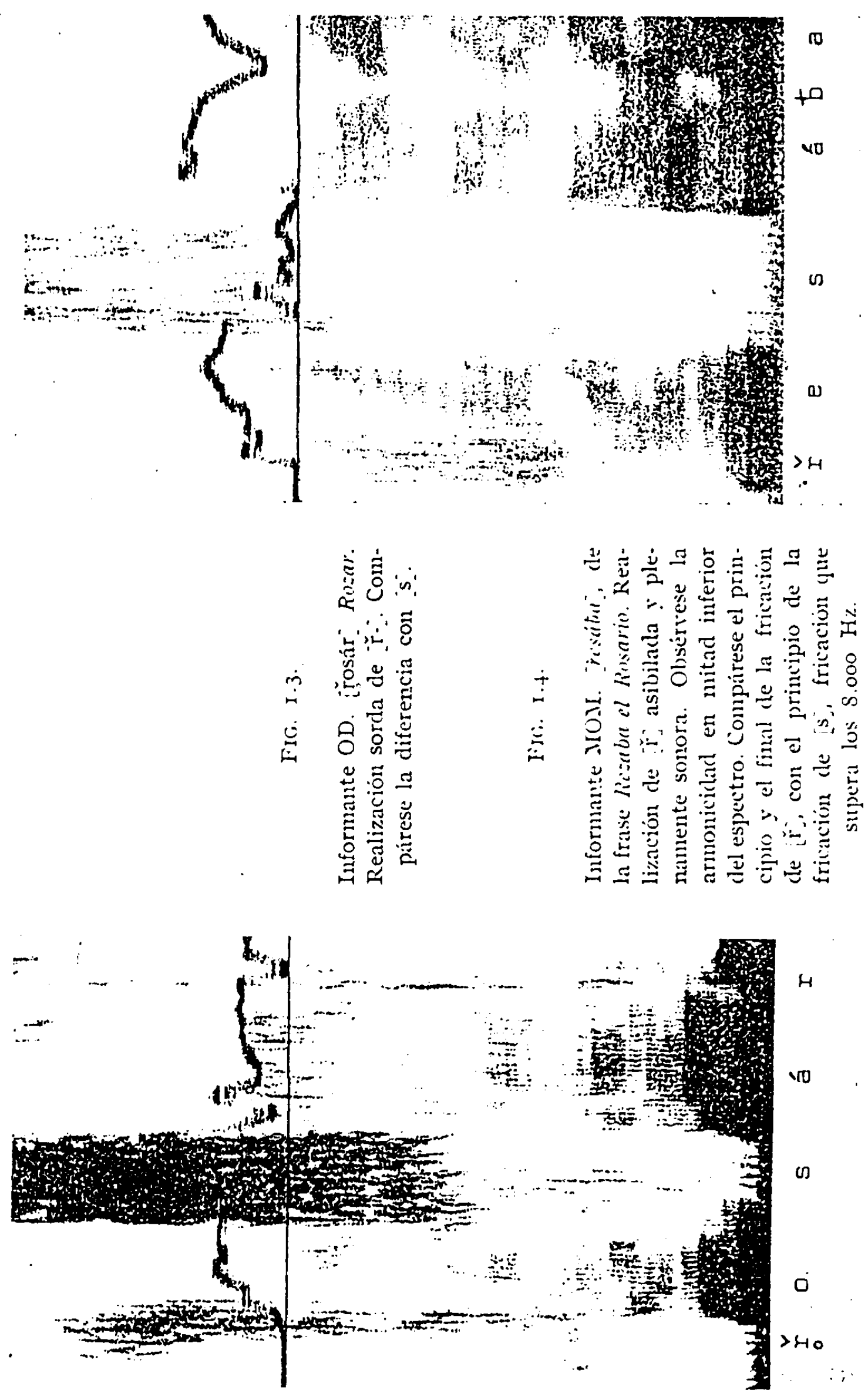
El $F_{2}$ de $/ 0 /$ está por debajo del $F_{2}$ de $[\check{r}]$ y su transición es $(+)$. El $F_{2}$ de $/ \mathrm{u} /$ está también por debajo del $\mathrm{F}_{2}$ de $[\hat{r}]$ y su transición es $(+)$, con la excepción de ruso de MOM, en donde aparece como (-).

Con /a/, las cosas varían un poco: $T_{2}$ es positiva cuando $F_{2}$ de $[\check{r}]$ está por encima de $\mathrm{I}_{2} / \mathrm{a} /$, pero es negativa cuando está por debajo.

7. La transición del $\mathrm{I}_{1}$ es siempre negativa.

2. $[-\check{I}-]$ en posición intervocálica.

Los datos obtenidos son los siguientes ${ }^{1}$ :

x. [plriko]

\begin{tabular}{|c|c|c|c|c|c|c|c|c|c|}
\hline \multirow[b]{2}{*}{ Inf. } & \multirow[b]{2}{*}{ I } & \multirow[b]{2}{*}{2} & \multirow[b]{2}{*}{3} & \multirow[b]{2}{*}{4} & \multirow[b]{2}{*}{5} & \multicolumn{2}{|c|}{6} & \multicolumn{2}{|c|}{7} \\
\hline & & & & & & $F_{1}$ & $F_{2}$ & $T_{1}$ & $T_{2}$ \\
\hline ROM & 8 & $4^{86}$ & $x \cdot 701$ & arm. & 1.741 .5 & $\begin{array}{l}283.5 \\
324\end{array}$ & $\begin{array}{l}2.146,5 \\
1.822,5\end{array}$ & - & - \\
\hline $\mathrm{RGM}$ & 14,7 & $4^{80}$ & 1.984 & inar. & 2.835 & $\begin{array}{l}283.5 \\
283.5\end{array}$ & $\begin{array}{l}3.078 \\
2.956,5\end{array}$ & - & - \\
\hline OD & ro & 486 & I.70I & arun. & 2.187 & $\begin{array}{l}324 \\
324\end{array}$ & $\begin{array}{l}2.349 \\
2.430\end{array}$ & - & - \\
\hline MOM & 12,2 & 364.5 & I. $74 \mathrm{I}, 5$ & inar. & 2.268 & $\begin{array}{l}324 \\
405\end{array}$ & $\begin{array}{l}2.511 \\
2.430\end{array}$ & - & - \\
\hline
\end{tabular}

2. [irila]

\begin{tabular}{|c|c|c|c|c|c|c|c|c|c|}
\hline \multirow[b]{2}{*}{ Inf. } & \multirow[b]{2}{*}{ I } & \multirow[b]{2}{*}{2} & \multirow[b]{2}{*}{3} & \multirow[b]{2}{*}{4} & \multirow[b]{2}{*}{5} & \multicolumn{2}{|c|}{6} & \multicolumn{2}{|c|}{7} \\
\hline & & & & & & $F_{1}$ & $F_{2}$ & $T_{1}$ & $T_{2}$ \\
\hline ROM & IO, I & 405 & I. 579 & inar. & 2.268 & $\begin{array}{l}202,5 \\
324\end{array}$ & $\begin{array}{l}2.227,5 \\
2.5 \times I\end{array}$ & - & - \\
\hline $\mathrm{RGM}$ & 18,2 & 526.5 & $2.146,5$ & inar. & $3.44^{2}$ & $\begin{array}{l}240 \\
324\end{array}$ & $\begin{array}{l}3.078 \\
3.240\end{array}$ & - & - \\
\hline OD & 13.3 & $4^{86}$ & I. $45^{8}$ & inar. & 2.025 & $\begin{array}{l}283.5 \\
324\end{array}$ & $\begin{array}{l}2.430 \\
2.794,5\end{array}$ & - & - \\
\hline MOM & 13.3 & 567 & r. 863 & inar. & $2.3^{8} 9.5$ & $\begin{array}{l}324 \\
364.5\end{array}$ & $\begin{array}{l}2.430 \\
2.511\end{array}$ & - & - \\
\hline
\end{tabular}

1 Iil contorno en el que se han situado las palabras de este epígrafe, así como las de los siguientes, es: Digo la palabra otra vez. 


\section{3. [sjefre]}

\begin{tabular}{|c|c|c|c|c|c|c|c|c|c|}
\hline \multirow{2}{*}{ Inf. } & \multirow{2}{*}{ I } & \multirow{2}{*}{2} & \multirow{2}{*}{3} & \multirow{2}{*}{4} & \multirow{2}{*}{5} & \multicolumn{2}{|c|}{.6} & \multicolumn{2}{|c|}{7} \\
\hline & & & & & & $\mathrm{IH}_{2}$ & $\dot{H}_{2}$ & $T_{1}$ & $T_{2}$ \\
\hline ROM & 7.3 & 445.5 & I. 498 & inar. & 2.106 & $\begin{array}{l}364,5 \\
324\end{array}$ & $\begin{array}{l}1.620 \\
1.377\end{array}$ & - & - \\
\hline RGM & II, 9 & 648 & $2.146,5$ & arm. & 2.997 & $\begin{array}{l}486 \\
486\end{array}$ & $\begin{array}{l}2.308 \\
2.187\end{array}$ & - & - \\
\hline$O D$ & 8,4 & 648 & r.70I & arm. & 2.349 & $\begin{array}{l}405 \\
405\end{array}$ & $\begin{array}{l}1.782 \\
1.70 x\end{array}$ & - & - \\
\hline MIOM & $10, x$ & $5^{67}$ & $89 \pi$ & arru. & 2.187 & $\begin{array}{l}480 \\
480\end{array}$ & $\begin{array}{l}1.863 \\
1.903 .5\end{array}$ & - & - \\
\hline
\end{tabular}

4. [ser êd]

\begin{tabular}{|c|c|c|c|c|c|c|c|c|c|}
\hline \multirow[b]{2}{*}{ Inf. } & \multirow[b]{2}{*}{ I } & \multirow[b]{2}{*}{2} & \multirow[b]{2}{*}{3} & \multirow[b]{2}{*}{4} & \multirow[b]{2}{*}{5} & \multicolumn{2}{|c|}{6} & \multicolumn{2}{|c|}{7} \\
\hline & & & & & & $1 i_{1}$ & $F_{2}$ & $T_{1}$ & $T_{2}$ \\
\hline ROAI & I I ,9 & 526,5 & I. 579 & inar. & I.863 & $\begin{array}{l}405 \\
405\end{array}$ & $\begin{array}{l}1.822 \\
2.025\end{array}$ & - & - \\
\hline RGMI & .14 .7 & 445.5 & 2.100 & inar. & 2.875 & $\begin{array}{l}\overrightarrow{405} \\
526.5\end{array}$ & $\begin{array}{l}2.430 \\
2.268\end{array}$ & - & - \\
\hline OD & I 6 & 648 & I.4I7 & arm. & 2.025 & $\begin{array}{l}445 \\
486\end{array}$ & $\begin{array}{l}2.025 \\
2.106\end{array}$ & - & - \\
\hline MIOM & $2 I$ & Sro & I.093.5 & arm. & 2.268 & $\begin{array}{l}445,5 \\
526,5\end{array}$ & $\begin{array}{l}2.106 \\
2.511\end{array}$ & - & - \\
\hline
\end{tabular}




\section{5. [púra]}

\begin{tabular}{|c|c|c|c|c|c|c|c|c|c|}
\hline \multirow{2}{*}{ Inf. } & \multirow{2}{*}{$\mathbf{I}$} & \multirow{2}{*}{2} & \multirow{2}{*}{3} & \multirow{2}{*}{4} & \multirow{2}{*}{5} & \multicolumn{2}{|c|}{6} & \multicolumn{2}{|c|}{7} \\
\hline & & & & & & $F_{1}$ & $I_{2}$ & $T_{1}$ & ' \\
\hline ROM & 8.4 & 364.5 & $1.984,5$ & arm. & 2.227 .5 & $\begin{array}{l}729 \\
526,5\end{array}$ & $\begin{array}{l}\text { r.377 } \\
\text { I.2I5 }\end{array}$ & - & - \\
\hline RGM & 12,6 & 364,5 & 2.065 .5 & inar. & 2.956 .5 & $\begin{array}{r}1.053 \\
891\end{array}$ & $\begin{array}{l}x .782 \\
x .620\end{array}$ & - & $\begin{array}{l}t \\
+\end{array}$ \\
\hline OD & 10,8 & 648 & $1.45^{8}$ & arm. & I.944 & $\begin{array}{l}89 r \\
93 r .5\end{array}$ & $\begin{array}{l}I .4 I 7.5 \\
I .4 I 7.5\end{array}$ & - & $\begin{array}{l}= \\
=\end{array}$ \\
\hline MOM & 14.7 & 364 & 972 & arm. & 2.268 & $\begin{array}{r}1.012 .5 \\
769.5\end{array}$ & $\begin{array}{l}1.74 \mathrm{I} .5 \\
\mathrm{r} .296\end{array}$ & - & - \\
\hline
\end{tabular}

\section{6. [par̃ál]}

\begin{tabular}{|c|c|c|c|c|c|c|c|c|c|}
\hline \multirow{2}{*}{ Inf. } & \multirow[b]{2}{*}{ I } & \multirow{2}{*}{2} & \multirow[b]{2}{*}{3} & \multirow[b]{2}{*}{4} & \multirow[b]{2}{*}{5} & \multicolumn{2}{|c|}{6} & \multicolumn{2}{|c|}{7} \\
\hline & & & & & & $F_{1}$ & $F_{2}$ & $T_{1}$ & $T_{2}$ \\
\hline ROM & I 3.6 & 405 & $\begin{array}{c}\mathbf{1} .822,5 \\
\end{array}$ & arm. & 2.187 & $\begin{array}{l}729 \\
810\end{array}$ & $\begin{array}{l}\text { I. } 4 \text { I } 7.5 \\
\text { I. } 579.5\end{array}$ & - & - \\
\hline RGM & 18,5 & 364.5 & 2.106 & inar. & 2.916 & $\begin{array}{l}\text { I.0I } 2,5 \\
\text { I.I } 34\end{array}$ & $\begin{array}{l}\text { I. } 822,5 \\
r .822,5\end{array}$ & $\begin{array}{l}= \\
=\end{array}$ & $\begin{array}{l}+ \\
+\end{array}$ \\
\hline OD & 12,9 & 648 & I. 498,5 & inar. & 1.620 & $\begin{array}{l}89 x \\
93 \pi, 5\end{array}$ & $\begin{array}{l}\text { I.377 } \\
\text { I. } 498.5\end{array}$ & - & - \\
\hline MOM & 13.3 & 688 & I.01 2,5 & arm. & 2.592 & $\begin{array}{c}810 \\
\mathrm{x} .012,5\end{array}$ & $\begin{array}{l}1.701 \\
1.863\end{array}$ & - & - \\
\hline
\end{tabular}




\section{7. [kóro]}

\begin{tabular}{|c|c|c|c|c|c|c|c|c|c|}
\hline \multirow{2}{*}{ Inf. } & \multirow{2}{*}{$\mathbf{I}$} & \multirow{2}{*}{2} & \multirow{2}{*}{3} & \multirow{2}{*}{4} & \multirow{2}{*}{5} & \multicolumn{2}{|c|}{6} & \multicolumn{2}{|c|}{7} \\
\hline & & & & & & $\mathrm{IP}_{1}$ & $I_{2}$ & $\mathrm{I}_{1}$ & 12 \\
\hline ROM & 8,4 & 445.5 & $x .053$ & armi. & $x .296$ & $\begin{array}{l}445,5 \\
364,5\end{array}$ & $\begin{array}{c}972 \\
\mathbf{r} .093 .5\end{array}$ & - & + \\
\hline RGMI & 14.7 & 405 & I. 498,5 & inar. & 2.592 & $\begin{array}{l}607,5 \\
648\end{array}$ & $\begin{array}{l}1.093 .5 \\
1.215\end{array}$ & $\begin{array}{l}= \\
=\end{array}$ & + \\
\hline OD & 7 & 688,5 & 1.174 .5 & inar. & $\mathbf{r . 4 9 8 . 5}$ & $\begin{array}{l}607.5 \\
567\end{array}$ & $\begin{array}{l}x .174,5 \\
\text { r.x } 34\end{array}$ & - & $\begin{array}{l}= \\
=\end{array}$ \\
\hline MOM & $9, \mathrm{I}$ & 364.5 & $1.012,5$ & arm. & I.296 & $\begin{array}{l}567 \\
526,5\end{array}$ & $\begin{array}{l}1.255 .5 \\
1.296\end{array}$ & - & - \\
\hline
\end{tabular}

8. [orór]

\begin{tabular}{|c|c|c|c|c|c|c|c|c|c|}
\hline \multirow{2}{*}{ Inf. } & \multirow[b]{2}{*}{$I$} & \multirow{2}{*}{2} & \multirow{2}{*}{3} & \multirow[b]{2}{*}{4} & \multirow[b]{2}{*}{5} & \multicolumn{2}{|c|}{6} & \multicolumn{2}{|c|}{7} \\
\hline & & & & & & $F_{1}$ & $r_{2}$ & $T_{1}$ & $\mathrm{~T}_{2}$ \\
\hline ROM & 9.8 & 364.5 & I. $\times 34$ & inar. & 1.377 & $\begin{array}{l}324 \\
364.5\end{array}$ & $\begin{array}{l}\text { I.OI } 2,5 \\
\text { I. or } 2,5\end{array}$ & - & $\begin{array}{l}+ \\
+\end{array}$ \\
\hline RGMI & $x 6,8$ & - & 1.620 & inar. & $2.51 \mathrm{I}$ & $\begin{array}{l}607.5 \\
607.5\end{array}$ & $\begin{array}{r}1.215 \\
x .215\end{array}$ & - & $\begin{array}{l}+ \\
+\end{array}$ \\
\hline OD & $I_{4}$ & 486 & $1.336,5$ & inar. & I. 539 & $\begin{array}{l}405 \\
567\end{array}$ & $\begin{array}{l}1.296 \\
1.377\end{array}$ & - & - \\
\hline MOM & $I x, 9$ & 526,5 & $x .093,5$ & arm. & I. 903,5 & $\begin{array}{l}4^{86} \\
567\end{array}$ & $\begin{array}{l}x .134 \\
1.336 .5\end{array}$ & - & $=$ \\
\hline
\end{tabular}




\section{9. [pớn $]$}

\begin{tabular}{|c|c|c|c|c|c|c|c|c|c|}
\hline \multirow{2}{*}{ Inf. } & \multirow{2}{*}{$\mathbf{I}$} & \multirow{2}{*}{2} & \multirow{2}{*}{3} & \multirow{2}{*}{4} & \multirow{2}{*}{5} & \multicolumn{2}{|c|}{6} & \multicolumn{2}{|c|}{7} \\
\hline & & & & & & $l_{1}$ & $\mathrm{I}_{2}$ & $T_{1}$ & $T_{2}$ \\
\hline $\mathrm{ROM}$ & 9,8 & 445.5 & 1.093 .5 & arm. & $x .4 \mathrm{I} 7.5$ & $\begin{array}{l}324 \\
324\end{array}$ & $\begin{array}{l}x .012,5 \\
1.012,5\end{array}$ & - & $\begin{array}{l}+ \\
+\end{array}$ \\
\hline $\mathrm{RGM}$ & II, 9 & 364,5 & I. $45^{8}$ & inar. & 2.673 & $\begin{array}{l}526,5 \\
486\end{array}$ & $\begin{array}{l}\text { I. } 296 \\
\text { I. } 2 \text { I } 5\end{array}$ & - & $\begin{array}{l}+ \\
+\end{array}$ \\
\hline OD & 10,8 & 486 & 1.215 & arm. & I. 782 & $\begin{array}{l}445.5 \\
445.5\end{array}$ & $\begin{array}{l}x .053 \\
1.093 .5\end{array}$ & - & $\begin{array}{l}+ \\
+\end{array}$ \\
\hline MOM & $\times 3.3$ & 567 & 1.053 & arm. & I.539 & $\begin{array}{l}405 \\
729\end{array}$ & $\begin{array}{l}\text { I.053 } \\
\text { I. } 296\end{array}$ & - & $=$ \\
\hline
\end{tabular}

Io. [óliro ]

\begin{tabular}{|c|c|c|c|c|c|c|c|c|c|}
\hline \multirow[b]{2}{*}{ Inf. } & \multirow[b]{2}{*}{$x$} & \multirow[b]{2}{*}{2} & \multirow[b]{2}{*}{3} & \multirow[b]{2}{*}{4} & \multirow[b]{2}{*}{5} & \multicolumn{2}{|c|}{6} & \multicolumn{2}{|c|}{7} \\
\hline & & & & & & $\mathrm{F}_{1}$ & $\mathrm{~F}_{2}$ & $T_{2}$ & $T_{2}$ \\
\hline ROM & 9,8 & 445.5 & 1:093 & arm. & 1.255 .5 & $\begin{array}{l}243 \\
243\end{array}$ & $\begin{array}{c}648 \\
1.090,5\end{array}$ & - & $\begin{array}{l}+ \\
=\end{array}$ \\
\hline RGM & 14,7 & 405 & I. 539 & inar. & $2.632,5$ & $\begin{array}{l}324 \\
607.5\end{array}$ & $\begin{array}{r}972 \\
\mathrm{I} \cdot 377\end{array}$ & $\begin{array}{l}= \\
+\end{array}$ & $\begin{array}{l}+ \\
+\end{array}$ \\
\hline OD & 6,6 & 648 & I.134 & arm. & $1.45^{8}$ & $\begin{array}{l}526,5 \\
526,5\end{array}$ & $\begin{array}{r}931.5 \\
\mathrm{r} .255,5\end{array}$ & - & + \\
\hline MOM & $\times 3.3$ & 405 & 972 & arm. & $\mathrm{r} .863$ & $\begin{array}{l}405 \\
688.5\end{array}$ & $\begin{array}{l}1.012,5 \\
1.377\end{array}$ & - & $=$ \\
\hline
\end{tabular}


II. [surón]

\begin{tabular}{|c|c|c|c|c|c|c|c|c|c|}
\hline \multirow{2}{*}{ Inf. } & \multirow{2}{*}{ I } & \multirow{2}{*}{2} & \multirow{2}{*}{3} & \multirow{2}{*}{4} & \multirow{2}{*}{5} & \multicolumn{2}{|c|}{6} & \multicolumn{2}{|c|}{7} \\
\hline & & & & & & $r_{1}$ & $I_{3}$ & $T_{2}$ & $\mathrm{~T}_{2}$ \\
\hline ROM & 10,8 & 364,5 & 1.255 .5 & inar. & 1.620 & $\begin{array}{l}283.5 \\
445.5\end{array}$ & $\begin{array}{r}93 \times, 5 \\
1.093 .5\end{array}$ & $=$ & $\begin{array}{l}+ \\
+\end{array}$ \\
\hline RGM & 12,6 & 405 & $1.45^{8}$ & arm. & 2.430 & $\begin{array}{l}324 \\
486\end{array}$ & $\begin{array}{l}1.296 \\
1.458\end{array}$ & - & $\begin{array}{l}+ \\
+\end{array}$ \\
\hline OD & $\times 3.3$ & 526,5 & 1.296 & inar. & 2.187 & $\begin{array}{l}243 \\
486\end{array}$ & $\begin{array}{r}648 \\
r .255 .5\end{array}$ & - & - \\
\hline MOM & 12,2 & 526,5 & I.I 74,5 & arm. & $2.308,5$ & $\begin{array}{l}364,5 \\
729\end{array}$ & $\begin{array}{l}1.296 \\
1.336,5\end{array}$ & - & - \\
\hline
\end{tabular}

12. [korér]

\begin{tabular}{|c|c|c|c|c|c|c|c|c|c|}
\hline \multirow{2}{*}{ Inf.' } & \multirow{2}{*}{ I } & \multirow{2}{*}{2} & \multirow{2}{*}{3} & \multirow{2}{*}{4} & \multirow{2}{*}{5} & \multicolumn{2}{|c|}{6} & \multicolumn{2}{|c|}{7} \\
\hline & & & & & & $F_{1}$ & $F_{2}$ & $T_{1}$ & $T_{2}$ \\
\hline RONI & 10,8 & 648 & I. 336,5 & inar. & 1.620 & $\begin{array}{l}405 \\
405\end{array}$ & $\begin{array}{l}\text { r. } 74,5 \\
\text { r.822,5 }\end{array}$ & - & + \\
\hline RGM & $\times 9,6$ & 526.5 & $x .822,5$ & inar. & 2.673 & $\begin{array}{l}567 \\
567\end{array}$ & $\begin{array}{l}1.215 \\
2.592\end{array}$ & - & + \\
\hline OD & $x 0,8$ & 526,5 & $x .336 .5$ & arm. & 1.620 & $\begin{array}{l}4^{86} \\
567\end{array}$ & $\begin{array}{l}\text { r. } 336,5 \\
2 . x 87\end{array}$ & - & - \\
\hline MOMI & $I I, 5$ & 526,5 & $93 r, 5$ & arm. & $x .984,5$ & $\begin{array}{l}405 \\
405\end{array}$ & $\begin{array}{l}1.215 \\
2.106\end{array}$ & - & - \\
\hline
\end{tabular}



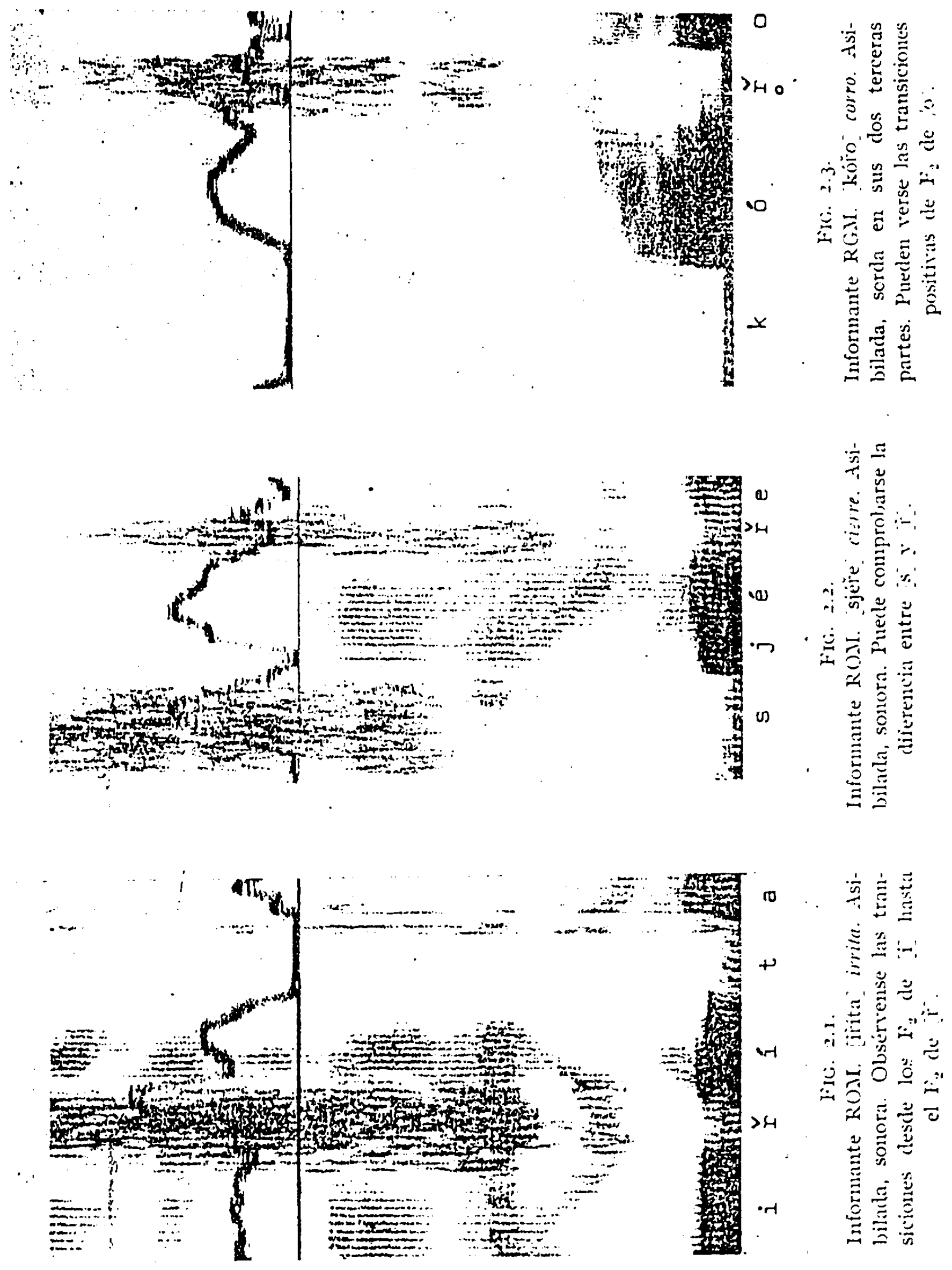

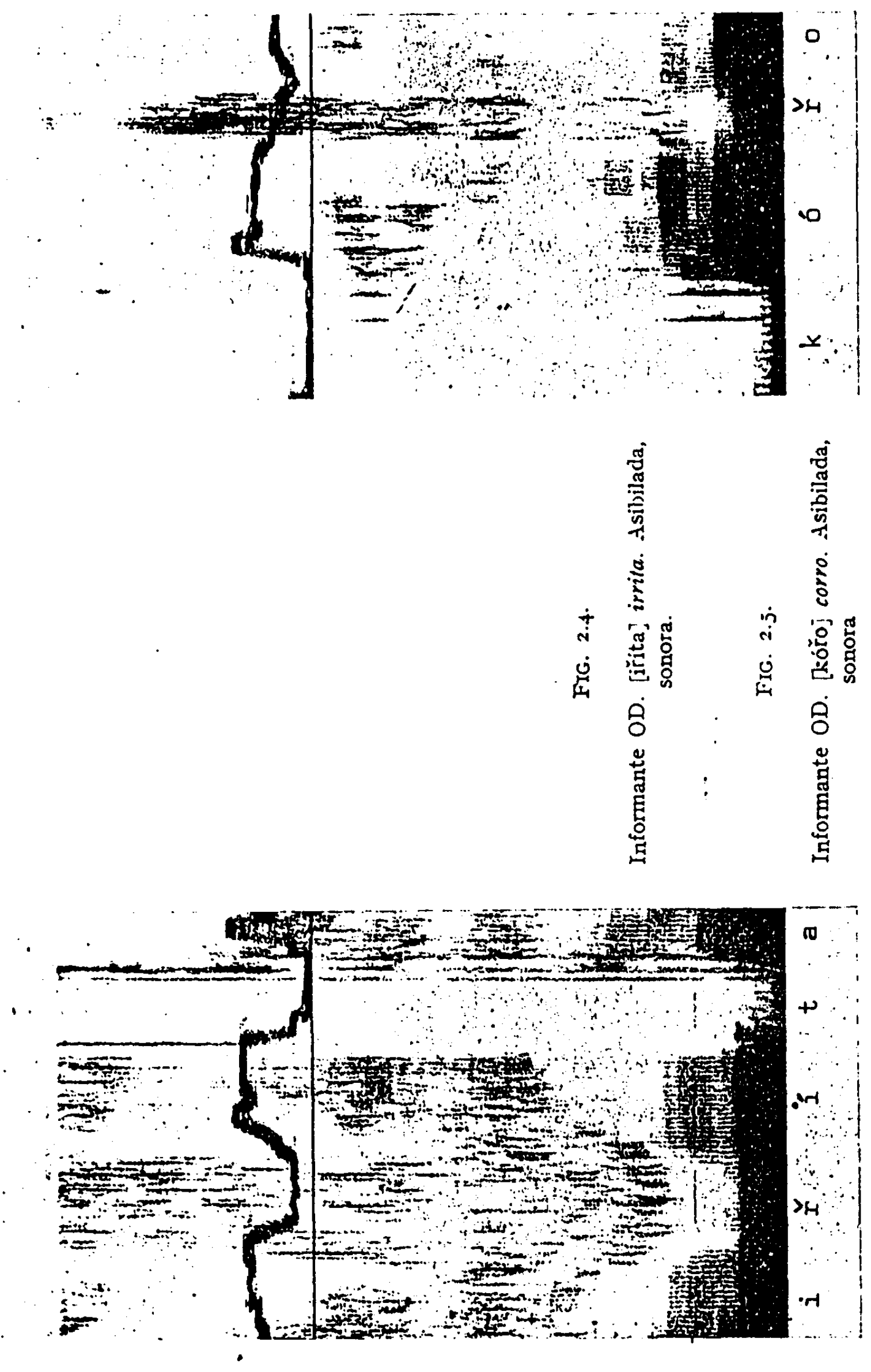

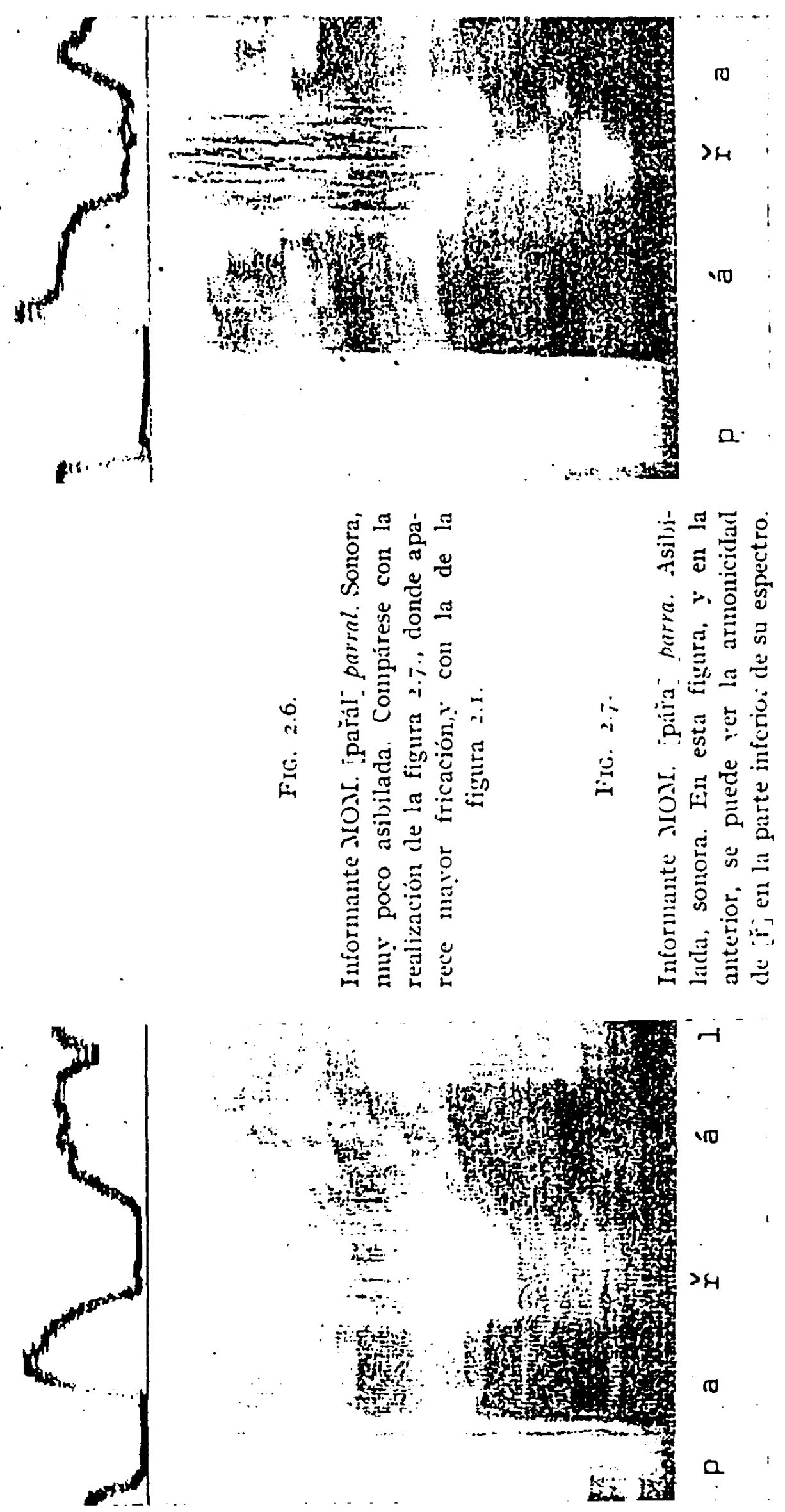


\begin{tabular}{|c|c|c|c|c|c|c|c|c|c|}
\hline \multirow{2}{*}{ Inf. } & \multirow{2}{*}{ I } & \multirow{2}{*}{2} & \multirow{2}{*}{3} & \multirow{2}{*}{4} & \multirow{2}{*}{5} & \multicolumn{2}{|c|}{6} & \multicolumn{2}{|c|}{7} \\
\hline & & & & & & $\mathrm{F}_{1}$ & $F_{2}$ & $T_{1}$ & $T_{1}$ \\
\hline ROM & ro & 405 & I.255.5 & inar. & 1.620 & $\begin{array}{l}364,5 \\
769,5\end{array}$ & $\begin{array}{r}972 \\
\mathrm{I} \cdot 336,5\end{array}$ & - & + \\
\hline $\mathrm{RGM}$ & 16,4 & - & $\mathrm{I} .822,5$ & inar. & 2.592 & $\begin{array}{c}648 \\
r .012,5\end{array}$ & $\begin{array}{l}1.215 \\
1.863\end{array}$ & + & $\begin{array}{l}+ \\
+\end{array}$ \\
\hline OD & 14 & 648 & 1.296 & arm. & 1.620 & $\begin{array}{l}405 \\
769\end{array}$ & $\begin{array}{l}1.053 \\
1.782\end{array}$ & - & + \\
\hline MOM & $x 2,2$ & 526 & I.0工 2.5 & arw. & I. 903.5 & $\begin{array}{l}445 \\
972\end{array}$ & $\begin{array}{l}\text { I.I74 } \\
\text { r.70I }\end{array}$ & - & - \\
\hline
\end{tabular}

\section{CONCLUSIONES}

Los resultados que obtenemos de los cuadros anteriores son:

I. La duración de [- $\breve{r}-]$ en sillaba tónica es de $x 8,19 \mathrm{cs}$; en sílaba átona, $12,2 \mathrm{cs}$.

2. De los 52 casos analizados, 42 son plenamente sonoros; 2 , realizaciorfes totalmente sordas (orror y forrar de RGM); 8 , realizaciones ensordecidas en su segunda parte o en su parte final (irrita, parra, parral, corro, burro, correr de RGM y cerré, zurrón de ROM).

El límite superior de $F_{1}$ en las realizaciones sonoras o en la parte :sonora de las ensordecidas alcanza una altura de $509,8 \mathrm{r} \mathrm{Hz}$ (en sílaba tónica la altura es algo mayor: $535.73 \mathrm{~Hz}$, mientras que en sílaba átona es de $483,9 \mathrm{~Hz}$ ).

3. La altura del $\mathrm{F}_{2}$ es la siguiente: con $/ \mathrm{i} /: \mathrm{I} .767,20 \mathrm{~Hz}$; con /e/: I.553,9 Hz; con /a/: r.6r4,93; con /o/: I.228,49; en el contorno /u-o/, ya que no hemos encontrado /u-u/: I.240,25 Hz. En los contornos /o-e/ y $/ 0-a /$ el valor de este $F_{2}$ se eleva, con relación a $/ u, o /$, alcanzando I.536,7 $\mathrm{Hz}$ y $\mathrm{I} .346,6 \mathrm{~Hz}$, respectivamente.

4. En 27 casos, la conformación del $\mathrm{F}_{2}$ es armónica, y en 25 inarmónica. Hemos observado que, cuando $\mathrm{F}_{2}$ es armónico, su nivel frecuencial es más bajo (valor medio de $1.289,9 \mathrm{~Hz}$ ) que cuando es inarmónico (valor medio, r.606,98 Hz). También las turbulencias de la fricación de $[\check{r}]$ comienzan a un nivel más bajo cuando $F_{2}$ es armónico (valor medio, r.950 Hz), y más alto cuando $\mathrm{F}_{2}$ es inarmónico (valor medio, $2.267,6 \mathrm{~Hz}$ ).

5. El comienzo de la fricación se produce a las siguientes frecuencias: con /i/: 2.393.5 Hz; con /e/: 2.333.75 Hz; con /a/: 2.338,87 Hz; 
con /o/: 1.785,33 Hz; en el contorno /u-0/: $1.969,3 \mathrm{~Hz}$. En el contorno /0-e/, la media es de $1.973,87 \mathrm{~Hz}$; en el contorno /0-a/: 1.933,87 $\mathrm{Hz}$.

6. La transición del $\mathrm{F}_{2}$ de las vocales adyacentes muestra los siguientes movimientos:

Al igual que en el caso anterior, el $\mathrm{F}_{2}$ de /i/ está a mayor frecuencia. que el $F_{2}$ de $\left[-\check{r}_{-}-\right], y$, por ello, $T_{2}$ es siempre negativo. Io mismo sucede $\operatorname{con} \mid \mathrm{e} /$.

Con $/ 0, u /$, el valor de $T_{2}$ varía: cuando el $F_{2}$ de [产] está por encima del segundo formante de las vocales contiguas, su transición es $(t)$; cuando está al mismo nivel, es horizontal $(=)$, y cuando está por debajo -en pocos casos- es negativa (-).

Con $/ a /$, las cosas varian bastante: no hemos visto una relación directa entre los valores de la transición de las vocales adyacentes y la posición del $\mathrm{I}_{2}$ de [r̂ $]$.

7. La transición del $i_{1}$ es, normalmente, negativa.

3. [r] en posición inicial de palabra, sin pausa precedente.

1. [paláora rima]

\begin{tabular}{|c|c|c|c|c|c|c|c|c|c|}
\hline \multirow{2}{*}{ Inf. } & \multirow{2}{*}{ I } & \multirow[b]{2}{*}{2} & \multirow[b]{2}{*}{3} & \multirow[b]{2}{*}{4} & \multirow[b]{2}{*}{5} & \multicolumn{2}{|c|}{6} & \multicolumn{2}{|c|}{7} \\
\hline & & & & & & $\mathrm{r}_{1}$ & $P_{2}$ & $\mathrm{~T}_{1}$ & $\mathrm{~T}_{2}$ \\
\hline ROM & 14 & 445,5 & $x .458$ & arm. & $1.74 \mathrm{I}, 5$ & $\begin{array}{l}526,5 \\
283,5\end{array}$ & $\begin{array}{l}1.417 .5 \\
2.632,5\end{array}$ & - & - \\
\hline RGM & 14 & 405 & 2.025 & inar. & 2.835 & $\begin{array}{c}1.053 \\
364.5\end{array}$ & $\begin{array}{l}\mathrm{r} .701 \\
3.078\end{array}$ & $\overline{-}$ & \pm \\
\hline$O D$ & 10 & 648 & I.336 & arm. & r. 782 & $\begin{array}{l}648 \\
243\end{array}$ & $\begin{array}{l}r .660,5 \\
2.227,5\end{array}$ & $\overline{-}$ & - \\
\hline MON & 16 & 526,5 & 931,5 & arm. & 2.187 & $\begin{array}{l}769,5 \\
364,5\end{array}$ & $\begin{array}{l}1.944 \\
2.794 .5\end{array}$ & $\overline{-}$ & $=$ \\
\hline
\end{tabular}




\section{2. [palábra Fisa]}

\begin{tabular}{|c|c|c|c|c|c|c|c|c|c|}
\hline \multirow[b]{2}{*}{ Inf. } & \multirow[b]{2}{*}{$\boldsymbol{I}$} & \multirow[b]{2}{*}{2} & \multirow[b]{2}{*}{3} & \multirow[b]{2}{*}{4} & \multirow[b]{2}{*}{5} & \multicolumn{2}{|c|}{6} & \multicolumn{2}{|c|}{7} \\
\hline & & & & & & $I_{1}$ & $1 i_{2}$ & $r_{1}$ & $T_{2}$ \\
\hline ROM & 13.6 & $4^{86}$ & $\mathrm{r} .4 \times 7.5$ & arm. & 2.025 & $\begin{array}{l}5^{67} \\
3^{24}\end{array}$ & $\begin{array}{l}x .620 \\
2.470 .5\end{array}$ & - & - \\
\hline RGM & 16 & 526,5 & 2.016 & inar. & 3.078 & $\begin{array}{l}648 \\
283.5\end{array}$ & $\begin{array}{l}2.025 \\
3.159\end{array}$ & - & $=$ \\
\hline OD & 12,6 & 526.5 & r. $45^{8}$ & inar. & 2.187 & $\begin{array}{l}850,5 \\
283,5\end{array}$ & $\begin{array}{l}\mathrm{I} .74 \mathrm{r}, 5 \\
2.673\end{array}$ & - & - \\
\hline MOM & 14,3 & $5^{67}$ & 810 & arm. & 2.430 & $\begin{array}{l}729 \\
243\end{array}$ & $\begin{array}{l}2.065 .5 \\
2.592\end{array}$ & - & - \\
\hline
\end{tabular}

\section{3. [palabra †éma]}

\begin{tabular}{|c|c|c|c|c|c|c|c|c|c|}
\hline \multirow[b]{2}{*}{ Inf. } & \multirow[b]{2}{*}{ I } & \multirow[b]{2}{*}{2} & \multirow[b]{2}{*}{3} & \multirow[b]{2}{*}{4} & \multirow[b]{2}{*}{5} & \multicolumn{2}{|c|}{6} & \multicolumn{2}{|c|}{7} \\
\hline & & & & & & $1 *_{1}$ & $I_{2}$ & $T_{1}$ & $T_{2}$ \\
\hline $120 \mathrm{M}$ & $\times 3,6$ & 480 & I.215 & arm. & $\mathrm{I} .74 \mathrm{r} .5$ & $\begin{array}{l}486 \\
364.5\end{array}$ & $\begin{array}{l}\text { I. } 336,5 \\
\text { I. } 984,5\end{array}$ & - & - \\
\hline RGM & $x 8,9$ & $3^{6}, 5$ & 2.106 & inar. & 2.835 & $\begin{array}{l}607.5 \\
364.5\end{array}$ & $\begin{array}{l}\text { r. } 782 \\
2.673\end{array}$ & - & + \\
\hline ON & $x 2,6$ & 445.5 & r.336.5 & arm. & I. 944 & $\begin{array}{l}648 \\
445.5\end{array}$ & $\begin{array}{l}x .620 \\
2.3^{8} 9.5\end{array}$ & - & - \\
\hline MOM & 16,4 & 486 & I. I 74.5 & arm. & $2.065,5$ & $\begin{array}{l}769.5 \\
567\end{array}$ & $\begin{array}{l}1.782 \\
1.863\end{array}$ & - & - \\
\hline
\end{tabular}


4. [paldora iesa]

\begin{tabular}{|c|c|c|c|c|c|c|c|c|c|}
\hline \multirow{2}{*}{ Inf. } & \multirow{2}{*}{$\mathbf{I}$} & \multirow[b]{2}{*}{2} & \multirow[b]{2}{*}{3} & \multirow[b]{2}{*}{4} & \multirow[b]{2}{*}{5} & \multicolumn{2}{|c|}{6} & \multicolumn{2}{|c|}{7} \\
\hline & & & & & & $F_{1}$ & $I_{2}$ & $T_{1}$ & $\mathrm{r}_{2}$ \\
\hline ROM & $I x, 5$ & 445,5 & 1.336 & inar. & 1.620 & $\begin{array}{l}567 \\
283,5\end{array}$ & $\begin{array}{l}1.45^{8} \\
2.065 .5\end{array}$ & - & - \\
\hline RGM & 17.5 & 526,5 & $2.794,5$ & inar. & 2.916 & $\begin{array}{l}567 \\
486\end{array}$ & $\begin{array}{l}2.065 .5 \\
2.7 \times 3.5\end{array}$ & - & - \\
\hline OD & 11,9 & 648 & 1.296 & inar. & 2.470 .5 & $\begin{array}{l}891 \\
526,5\end{array}$ & $\begin{array}{l}1.741 .5 \\
2.470,5\end{array}$ & - & - \\
\hline MOMI & $x_{4}, 7$ & 567 & I. 134 & arm. & $2.470,5$ & $\begin{array}{l}89 x \\
405\end{array}$ & $\begin{array}{l}1.863 \\
2.268\end{array}$ & - & - \\
\hline
\end{tabular}

5. [palábra fli]

\begin{tabular}{|c|c|c|c|c|c|c|c|c|c|}
\hline \multirow{2}{*}{ Iuf. } & \multirow{2}{*}{ I } & \multirow{2}{*}{2} & \multirow{2}{*}{3} & \multirow{2}{*}{4} & \multirow{2}{*}{5} & \multicolumn{2}{|c|}{6} & \multicolumn{2}{|c|}{7} \\
\hline & & & & & & $\mathrm{I}_{1}$ & $\mathrm{I}_{3}$ & $T_{1}$ & $T_{2}$ \\
\hline ROMI & 9,4 & 405 & 1.417 .5 & inar. & $\mathbf{x} .865$ & $\begin{array}{l}769,5 \\
526,5\end{array}$ & $\begin{array}{l}I .498,5 \\
1.863\end{array}$ & - & - \\
\hline RGM & 19,9 & 567 & $2 . \pi 46,5$ & inar. & 2.916 & $\begin{array}{l}648 \\
607,5\end{array}$ & $\begin{array}{l}x .984 .5 \\
2.3^{8} 9.5\end{array}$ & - & + \\
\hline OD & 12,9 & 607,5 & 1.417 .5 & arm. & 2.106 & $\begin{array}{l}688,5 \\
445,5\end{array}$ & $\begin{array}{l}1.660,5 \\
2.065 .5\end{array}$ & - & - \\
\hline MOA & 15.5 & 567 & I.21 5 & arm. & 2.673 & $\begin{array}{l}810 \\
405\end{array}$ & $\begin{array}{l}1.782 \\
2.146,5\end{array}$ & - & - \\
\hline
\end{tabular}




\section{6. [paláora remár]}

\begin{tabular}{|c|c|c|c|c|c|c|c|c|c|}
\hline \multirow{2}{*}{ Inf. } & \multirow{2}{*}{ I } & \multirow{2}{*}{2} & \multirow[b]{2}{*}{3} & \multirow[b]{2}{*}{4} & \multirow[b]{2}{*}{5} & \multicolumn{2}{|c|}{6} & \multicolumn{2}{|c|}{7} \\
\hline & & & & & & $l_{1}$ & $1 ; 2$ & $T_{1}$ & 'T, \\
\hline ROM & 8.5 & 405 & $x .427,5$ & inar. & I. 863 & $\begin{array}{l}648 \\
364,5\end{array}$ & $\begin{array}{l}x .45^{8} \\
x .74 I, 5\end{array}$ & - & - \\
\hline RGM & 12,2 & 567 & $2.146,5$ & inar. & 3.078 & $\begin{array}{l}607.5 \\
364.5\end{array}$ & $\begin{array}{l}1.74 I, 5 \\
2.551,5\end{array}$ & - & + \\
\hline OD & 9,1 & 648 & $1.45^{8}$ & arm. & 2.754 & $\begin{array}{l}688.5 \\
445.5\end{array}$ & $\begin{array}{l}1.70 x \\
1.620\end{array}$ & - & - \\
\hline MOM & $I X, 9$ & 567 & 1.053 & arm. & $2.146,5$ & $\begin{array}{l}769.5 \\
405\end{array}$ & $\begin{array}{l}x .782 \\
x .984 .5\end{array}$ & - & - \\
\hline
\end{tabular}

7. [paiábra łás]

\begin{tabular}{|c|c|c|c|c|c|c|c|c|c|}
\hline \multirow{2}{*}{ Inf. } & \multirow[b]{2}{*}{$\mathbf{I}$} & \multirow[b]{2}{*}{2} & \multirow[b]{2}{*}{3} & \multirow[b]{2}{*}{4} & \multirow[b]{2}{*}{5} & \multicolumn{2}{|c|}{6} & \multicolumn{2}{|c|}{7} \\
\hline & & & & & & $F_{1}$ & $F_{3}$ & $T_{1}$ & $T_{2}$ \\
\hline ROM & 12,6 & 526,5 & 1.620 & inar. & x. 863 & $\begin{array}{l}526,5 \\
810\end{array}$ & $\begin{array}{l}\text { I. } 45^{8} \\
\text { I.498.5 }\end{array}$ & - & - \\
\hline $\mathrm{RGM}$ & 17,8 & $5^{67}$ & I. 984,5 & inar. & 2.916 & $\begin{array}{l}567 \\
850,5\end{array}$ & $\begin{array}{l}2.065 .5 \\
1.741 .5\end{array}$ & - & $\begin{array}{l}+ \\
+\end{array}$ \\
\hline OD & 12,2 & 648 & 1.296 & arm. & 2.106 & $\begin{array}{l}850,5 \\
891\end{array}$ & $\begin{array}{l}\text { I. } 600,5 \\
\text { I. } 45^{8}\end{array}$ & - & - \\
\hline MOAI & 14.7 & 526,5 & I.01 2,5 & arm. & 2.308 .5 & $\begin{array}{l}850,5 \\
972\end{array}$ & $\begin{array}{l}1.782 \\
x .701\end{array}$ & - & - \\
\hline
\end{tabular}




\section{8. [paldora rása]}

\begin{tabular}{|c|c|c|c|c|c|c|c|c|c|}
\hline \multirow{2}{*}{ Inf. } & \multirow{2}{*}{ I } & \multirow{2}{*}{2} & \multirow{2}{*}{3} & \multirow{2}{*}{4} & \multirow[b]{2}{*}{5} & \multicolumn{2}{|c|}{6} & \multicolumn{2}{|c|}{7} \\
\hline & & & & & & $i_{1}$ & $1 i_{3}$ & $T_{1}$ & $\mathrm{~T}_{2}$ \\
\hline ROM & 13.5 & 445.5 & 1.215 & arm. & $x .660,5$ & $\begin{array}{l}688,5 \\
729\end{array}$ & $\begin{array}{l}x .4 \times 7.5 \\
x .377\end{array}$ & - & - \\
\hline RGM & 20,5 & $4^{86}$ & 1.863 & inar. & 2.835 & $\begin{array}{l}567 \\
769,5\end{array}$ & $\begin{array}{r}1.863 \\
x .782\end{array}$ & - & - \\
\hline OD & 12,2 & 648 & $x \cdot 377$ & aruu. & 1.620 & $\begin{array}{l}648 \\
891\end{array}$ & $\begin{array}{l}\text { I. } 498,5 \\
\text { I. } 498,5\end{array}$ & - & - \\
\hline MOM & 12,6 & 526,5 & $1.012,5$ & arm. & 2.349 & $\begin{array}{l}93 x, 5 \\
972\end{array}$ & $\begin{array}{l}1.782 \\
1.620\end{array}$ & - & - \\
\hline
\end{tabular}

9. [pálabra ráma]

\begin{tabular}{|c|c|c|c|c|c|c|c|c|c|}
\hline \multirow{2}{*}{ Inf. } & \multirow{2}{*}{ I } & \multirow{2}{*}{2} & \multirow[b]{2}{*}{3} & \multirow[b]{2}{*}{4} & \multirow[b]{2}{*}{5} & \multicolumn{2}{|c|}{6} & \multicolumn{2}{|c|}{$\cdot 7$} \\
\hline & & & & & & $\mathrm{F}_{1}$ & $i_{2}$ & $\mathrm{~T}_{1}$ & $\mathrm{~T}_{2}$ \\
\hline ROM & 13,6 & 364,5 & 1.701 & arm. & 2.187 & $\begin{array}{l}607,5 \\
688,5\end{array}$ & $\begin{array}{l}I .45^{8} \\
\text { I. } 4^{I} 7.5\end{array}$ & - & - \\
\hline RGM & 17,5 & 769.5 & I.944 & arm. & 2.835 & $\begin{array}{r}93 \times, 5 \\
x .093,5\end{array}$ & $\begin{array}{l}2.025 \\
1.782\end{array}$ & $\bar{t}$ & - \\
\hline OD & II,9 & $4^{86}$ & $1.336,5$ & arm. & x. $7^{82}$ & $\begin{array}{l}688,5 \\
729\end{array}$ & $\begin{array}{l}\mathbf{r} .45^{8} \\
\text { x.296 }\end{array}$ & - & - \\
\hline MOM & 14,7 & 567 & I.053 & arm. & 2.430 & $\begin{array}{r}769.5 \\
\times .012 .5\end{array}$ & $\begin{array}{l}r .822,5 \\
r .741,5\end{array}$ & - & - \\
\hline
\end{tabular}


ro. [palciora fos]

\begin{tabular}{|c|c|c|c|c|c|c|c|c|c|}
\hline \multirow{2}{*}{ Inf. } & \multirow{2}{*}{$\mathbf{I}$} & \multirow{2}{*}{2} & \multirow{2}{*}{3} & \multirow{2}{*}{4} & \multirow{2}{*}{5} & \multicolumn{2}{|c|}{6} & \multicolumn{2}{|c|}{7} \\
\hline & & & & & & $\mathrm{I}_{1}$ & $\mathrm{I}_{2}$ & $T_{1}$ & $\mathrm{~T}_{2}$ \\
\hline ROM & $I I, 2$ & 445.5 & $x .4 \times 7.5$ & arm. & $2.146,5$ & $\begin{array}{l}648 \\
364,5\end{array}$ & $\begin{array}{l}x .4 \times 7.5 \\
x .012,5\end{array}$ & - & $\bar{t}$ \\
\hline RGM & 18,9 & 729 & I.539 & inar. & 2.551 .5 & $\begin{array}{l}486 \\
526,5\end{array}$ & $\begin{array}{l}x .782 \\
x .093 .5\end{array}$ & - & $\overline{+}$ \\
\hline OD & 9.4 & 567 & I.296 & inar. & 2.106 & $\begin{array}{l}769.5 \\
526.5\end{array}$ & $\begin{array}{l}\text { I.539 } \\
\text { r.r } 74.5\end{array}$ & - & $\overline{+}$ \\
\hline MOM & 14,7 & 567 & 1.053 & arm. & I.903.5 & $\begin{array}{l}850,5 \\
486\end{array}$ & $\begin{array}{l}r .822 .5 \\
r .255 .5\end{array}$ & - & - \\
\hline
\end{tabular}

\section{I. [palábra řósa]}

\begin{tabular}{|c|c|c|c|c|c|c|c|c|c|}
\hline \multirow{2}{*}{ Inf. } & \multirow{2}{*}{$\mathbf{I}$} & \multirow{2}{*}{2} & \multirow{2}{*}{3} & \multirow[b]{2}{*}{4} & \multirow{2}{*}{5} & \multicolumn{2}{|c|}{6} & \multicolumn{2}{|c|}{7} \\
\hline & & & & & & $F_{1}$ & $F_{2}$ & $T_{1}$ & $T_{2}$ \\
\hline ROM & II,9 & 405 & $\mathrm{I} .498,5$ & inar. & 1.539 & $\begin{array}{l}5^{26}, 5 \\
324\end{array}$ & $\begin{array}{l}I .4 I 7.5 \\
\text { I.053 }\end{array}$ & - & + \\
\hline RGM & 19,2 & 567 & I. 498,5 & inar. & $2.470,5$ & $\begin{array}{l}607.5 \\
567\end{array}$ & $\begin{array}{l}x, 863 \\
x .093,5\end{array}$ & - & + \\
\hline OD & $I I, 2$ & 567 & $x .377$ & inar. & 2.025 & $\begin{array}{l}850,5 \\
526,5\end{array}$ & $\begin{array}{l}I .660,5 \\
I .2 I x, 5\end{array}$ & - & + \\
\hline MOM & 11,9 & 526,5 & 1.053 & arm. & 1.944 & $\begin{array}{l}769.5 \\
445.5\end{array}$ & $\begin{array}{l}\text { I.782 } \\
\text { I.I } 74,5\end{array}$ & - & - \\
\hline
\end{tabular}

6. -II 
12. [paldora forma]

\begin{tabular}{|c|c|c|c|c|c|c|c|c|c|}
\hline \multirow{2}{*}{ Inf. } & \multirow[b]{2}{*}{$\mathbf{I}$} & \multirow[b]{2}{*}{2} & \multirow[b]{2}{*}{3} & \multirow[b]{2}{*}{4} & \multirow[b]{2}{*}{5} & \multicolumn{2}{|c|}{6} & \multicolumn{2}{|c|}{7} \\
\hline & & & & & & $\mathrm{r}_{1}$ & $P_{8}$ & $\mathrm{~T}_{2}$ & $x$ \\
\hline ROM & 13,6 & 526,5 & 1.093 & arm. & I.377 & $\begin{array}{l}526,5 \\
486\end{array}$ & $\begin{array}{r}\mathrm{I} .45^{8} \\
93 x, 5\end{array}$ & - & + \\
\hline RGM & $x 7,1$ & 648 & $1.660,5$ & inar. & $2.55 \mathrm{r} .5$ & $\begin{array}{l}810 \\
405\end{array}$ & $\begin{array}{c}\pi \cdot 74 \mathrm{r}, 5 \\
8 \mathrm{ro}\end{array}$ & $\overrightarrow{+}$ & + \\
\hline OD & 12,6 & 607.5 & $1.4 \times 7.5$ & inar. & $x . x 74.5$ & $\begin{array}{l}972 \\
567\end{array}$ & $\begin{array}{l}1.620 \\
1.2 \times 5\end{array}$ & - & $=$ \\
\hline MOM & 16,8 & $4^{86}$ & 972 & arm. & $\mathbf{I . 8 2 2 , 5}$ & $\begin{array}{l}891 \\
486\end{array}$ & $\begin{array}{l}x .782 \\
1.215\end{array}$ & - & $\dot{-}$ \\
\hline
\end{tabular}

13. [paláora fosár]

\begin{tabular}{|c|c|c|c|c|c|c|c|c|c|}
\hline \multirow[b]{2}{*}{ Inf. } & \multirow[b]{2}{*}{$\mathbf{I}$} & \multirow[b]{2}{*}{2} & \multirow[b]{2}{*}{3} & \multirow[b]{2}{*}{4} & \multirow[b]{2}{*}{5} & \multicolumn{2}{|c|}{6} & \multicolumn{2}{|c|}{7} \\
\hline & & & & & & $F_{1}$ & $F_{2}$ & $T_{1}$ & 1 \\
\hline ROMI & 7.7 & 405 & I. 174,5 & arm. & $x .4 \times 7.5$ & $\begin{array}{l}526,5 \\
283.5\end{array}$ & $\begin{array}{l}x .377 \\
x .093 .5\end{array}$ & - & $\bar{t}$ \\
\hline RGM & 10.5 & 324 & I. 498,5 & inar. & 2.430 & $\begin{array}{l}526,5 \\
405\end{array}$ & $\begin{array}{l}x .620 \\
1.215\end{array}$ & - & + \\
\hline OD & 7.3 & 607.5 & $1.093,5$ & arm. & 2.106 & $\begin{array}{l}769.5 \\
445.5\end{array}$ & $\begin{array}{l}r .45^{8} \\
1.093,5\end{array}$ & - & $=$ \\
\hline MOM & 7.7 & 526,5 & 972 & arm. & $1.660,5$ & $\begin{array}{l}769,5 \\
405\end{array}$ & $\begin{array}{l}\text { r.70r } \\
\text { r.or2.5 }\end{array}$ & - & - \\
\hline
\end{tabular}




\section{r4. [palábra ruso]}

\begin{tabular}{|c|c|c|c|c|c|c|c|c|c|}
\hline \multirow{2}{*}{ Inf. } & \multirow{2}{*}{$x$} & \multirow{2}{*}{2} & \multirow[b]{2}{*}{3} & \multirow[b]{2}{*}{4} & \multirow[b]{2}{*}{5} & \multicolumn{2}{|c|}{6} & \multicolumn{2}{|c|}{7} \\
\hline & & & & & & $I_{1}$ & $\mathbf{P}_{2}$ & $\mathrm{~T}_{1}$ & $T_{2}$ \\
\hline ROM & 9, I & 445.5 & 1.093 .5 & arin. & I.377 & $\begin{array}{l}567 \\
283.5\end{array}$ & $\begin{array}{r}r .4 \mathrm{I} 7.5 \\
850,5\end{array}$ & - & + \\
\hline RGM & $x_{7,8}$ & 526,5 & 1.620 & tuar. & 2.470 .5 & $\begin{array}{l}729 \\
324\end{array}$ & $\begin{array}{l}1.903 .5 \\
1.134\end{array}$ & - & + \\
\hline OD & 9,8 & 567 & I.255.5 & inar. & I. 498,5 & $\begin{array}{l}89 x \\
283,5\end{array}$ & $\begin{array}{l}1.45^{8} \\
\text { I. } 134\end{array}$ & - & - \\
\hline MOM & 14.7 & 526.5 & 810 & arm. & x.539 & $\begin{array}{l}769.5 \\
364,5\end{array}$ & $\begin{array}{c}1.822,5 \\
97^{2}\end{array}$ & - & - \\
\hline
\end{tabular}

\section{5. [palábra řmmjár]}

\begin{tabular}{|c|c|c|c|c|c|c|c|c|c|}
\hline \multirow{2}{*}{ Inf. } & \multirow{2}{*}{ I } & \multirow{2}{*}{2} & \multirow{2}{*}{3} & \multirow[b]{2}{*}{4} & \multirow[b]{2}{*}{5} & \multicolumn{2}{|c|}{6} & \multicolumn{2}{|c|}{7} \\
\hline & & & & & & $F_{1}$ & $F_{2}$ & $T_{1}$ & $T_{2}$ \\
\hline ROM & $9, \mathrm{r}$ & 445.5 & I.I34 & arm. & $x .4 \times 7.5$ & $\begin{array}{l}648 \\
324\end{array}$ & $\begin{array}{c}I .4 \mathrm{I} 7.5 \\
89 \mathrm{I}\end{array}$ & - & + \\
\hline RGM & $x 2,6$ & 526,5 & I. 579.5 & inar. & $2.470,5$ & $\begin{array}{l}607.5 \\
324\end{array}$ & $\begin{array}{l}\text { r.822,5 } \\
\text { r.134 }\end{array}$ & - & $\begin{array}{l}+ \\
+\end{array}$ \\
\hline OD & 8,4 & 486 & I. 336,5 & inar. & $\mathrm{r} .903 .5$ & $\begin{array}{l}89 \pi \\
405\end{array}$ & $\begin{array}{r}1.498,5 \\
850,5\end{array}$ & - & $\overline{+}$ \\
\hline MOM & 9.4 & 526.5 & $1.093,5$ & arm. & I. $74 \mathrm{I}, 5$ & $\begin{array}{l}648 \\
364.5\end{array}$ & $\begin{array}{l}1.701 \\
1.053\end{array}$ & - & + \\
\hline
\end{tabular}




\section{CONCI,USIONES}

I. La duración de [Y̌] en silaba tónica es de $\mathbf{x} 3,93$ cs; mientras que en silaba átona es de $9,53 \mathrm{cs}$.

2. Liste grupo comprende 60 realizaciones; de ellas, II están ensordecidas (rema, rey, remar, ras, rasa, ros, rosa, roma, ruso, rumiar de RGM y ras de ROM). La altura media que alcanza en su parte superior el $F_{1}$ es de $525,82 \mathrm{~Hz}$.

3. Los valores medios del $\mathrm{F}_{2}$ de [î] son: con $/ \mathrm{i} /: \mathbf{I} \cdot 442,75 \mathrm{~Hz}$; con . $\mathrm{e} /:$ I.54I, $5 \mathrm{~Hz}$; con /a/: I.45I,25 Hz; con /o/: I.288 Hz; con /u/: I.240, $3 \mathrm{I}$ $\mathrm{Hz}$.

4. $F_{2}$ aparece como armónico en 34 casos, y como inarmónico, en 26. Cuando es armónico, aparece a menor frecuencia (valor medio, $1.201,87 \mathrm{~Hz}$ ) que cuando es inarmónico (valor medio, $1.665,15 \mathrm{~Hz}$ ). Del mismo modo, cuando es armónico, las turbulencias de la fricación de [r̆] se producen a un nivel frecuencial más bajo $(1.992,85 \mathrm{~Hz}$ de valor medio) que cuando es inarmónico (valor medio $2.325,78 \mathrm{~Hz}$ ).

5. El comienzo de la fricación de [ř] se produce a las siguientes frecuencias: con /i/, a 2.283, $2 \mathrm{~Hz}$; con /e/, a 2.34I,5 Hz; con /a/, a 2.24 I Hz; .con $/ 0 /$, a $\mathrm{I} .95 \mathrm{r}, 5 \mathrm{~Hz}$; con $/ \mathrm{u} /$, a $\mathrm{I.802,2} \mathrm{Hz}$.

6. En los casos analizados, las transiciones del $F_{2}$ de la $[-a]$ de [palábra] son casi siempre negativas. (Unicamente el informante RGM ha dado cinco valores positivos - rima, rema, rey, remar, ras- y tres horizontales - risa, ruso, rumiar.)

Las transiciones del segundo formante de $/ \mathrm{i}, \mathrm{e} /$ son fuertemente

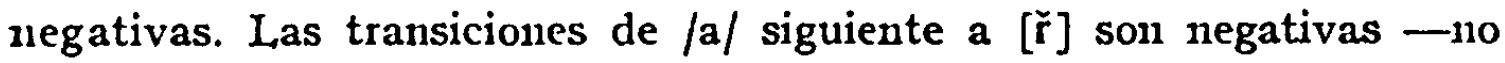
demasiado acusadas-, excepto en un caso: ras, de RGM, débilmente positiva. Las transiciones de $/ 0 /$ son positivas cuando su $F_{2}$ está por debajo del $\mathrm{F}_{2}$ de [ŕ] y negativas en caso contrario (excepto en Roma y rozar, de OD, que son horizontales). Las transiciones de / $u$ / cumplen las mismas condiciones que las de $/ 0 /$ (excepto en ruso, de OD, que es horizontal).

Las transiciones del primer formante son negativas, con dos excepciones, en que aparecen como positivas: rama y Roma, de RGM. 


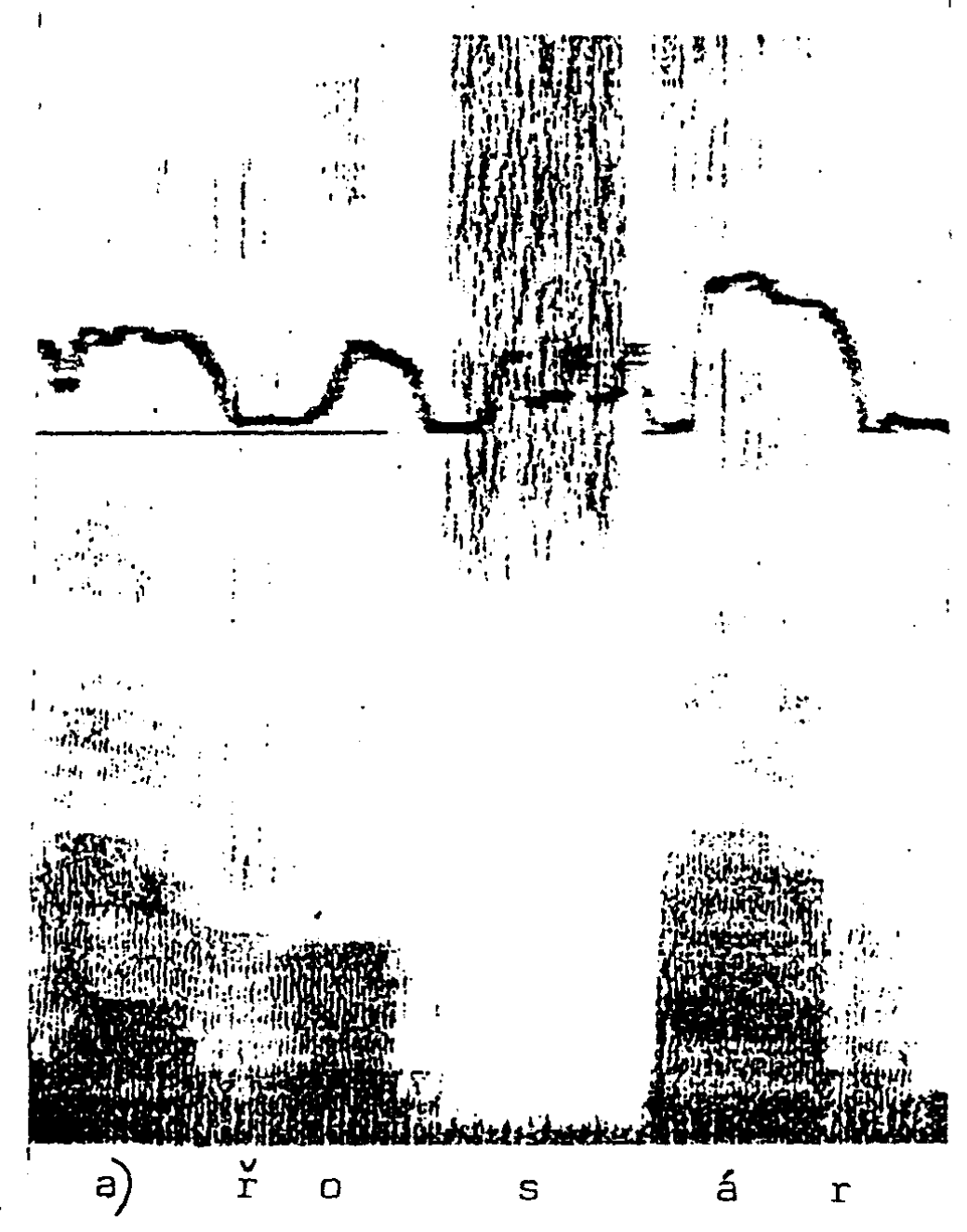

lill: 3.I.

Informante MUN!. frosár] de "palalura rosarw. Asibilatla. sonora, com fricalcion poco tensal (vease la linea de intensidlatl). Compratese la diferencia cont $[s]$. 


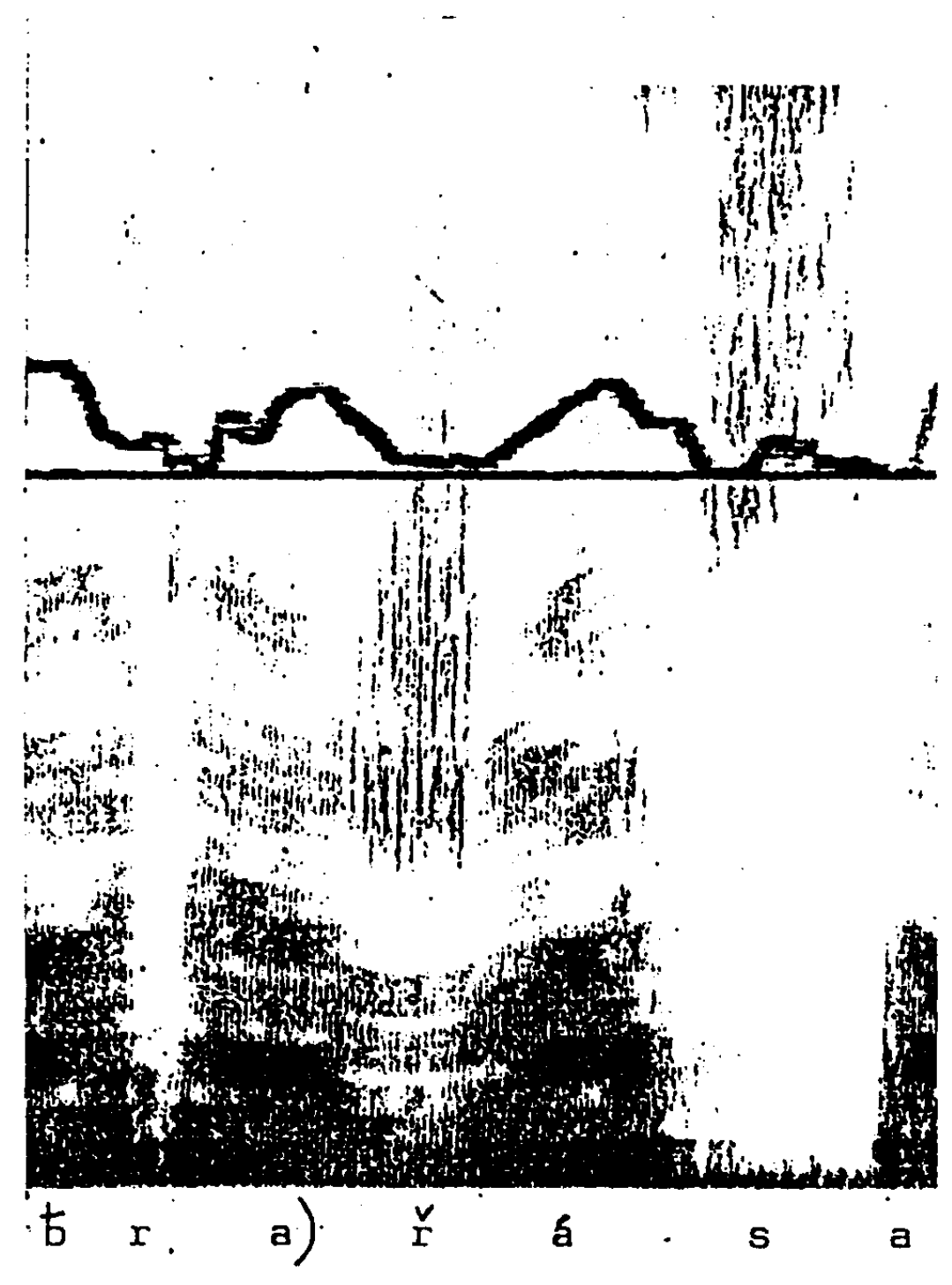

lisc. 3.2.

Informante MIOM. [ìisa] cle palabra rasan. Sonora, con más fricación que la cle la fig. 3.1. Muy armónica en su parte inferior. Obsérvese el principio y fin de la fricación. Confróntese con el principio de la fricación de [s], cuyo final supera los $\mathbf{8 . 0 0 0 ~} \mathbf{H z}$ del espectro. 
4. [г̃ precedido de [s].

I. $\mid$ los îsos/.

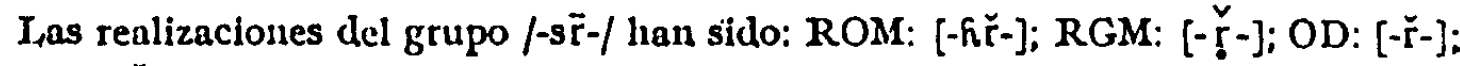
MOM: $[-\hat{h} \check{r}-]$.

\begin{tabular}{|c|c|c|c|c|c|c|c|c|c|}
\hline \multirow[b]{2}{*}{ Inf. } & \multirow[b]{2}{*}{ I } & \multirow{2}{*}{2} & \multirow[b]{2}{*}{3} & \multirow[b]{2}{*}{4} & \multirow[b]{2}{*}{5} & \multicolumn{2}{|c|}{6} & \multicolumn{2}{|c|}{7} \\
\hline & & & & & & $F_{1}$ & $F_{2}$ & $T_{1}$ & $T_{2}$ \\
\hline ROM & 11,9 & 526,5 & 1.296 & Brm. & 1.620 & 283.5 & $2.308,5$ & - & - \\
\hline RGM & 16,4 & - & 1.822 .5 & inar. & 2.835 & 283.5 & 3.199 .5 & 一 & - \\
\hline OD & 14 & $4^{86}$ & $1.45^{8}$ & inar. & 2.025 & 445.5 & 3.078 & - & - \\
\hline MOAI & 10,8 & 567 & I. $45^{8}$ & arm. & I. 74 I. 5 & 324 & 2.794 .5 & - & 一 \\
\hline
\end{tabular}

2. |los iémos/

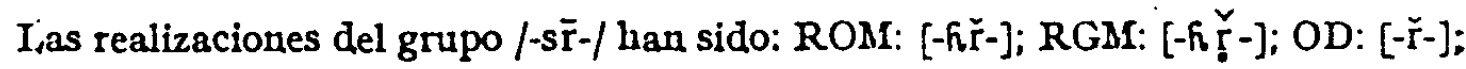
MOM: [-h̆r-].

\begin{tabular}{|c|c|c|c|c|c|c|c|c|c|}
\hline \multirow{2}{*}{ Inf. } & \multirow[b]{2}{*}{ I } & \multirow{2}{*}{2} & \multirow{2}{*}{3} & \multirow[b]{2}{*}{4} & \multirow[b]{2}{*}{5} & \multicolumn{2}{|c|}{6} & \multicolumn{2}{|c|}{7} \\
\hline & & & & & & $\mathrm{F}_{1}$ & $P_{2}$ & $T_{i}$ & $T_{2}$ \\
\hline ROM & . 9,8 & 324 & I.I 74.5 & arm. & I. 498,5 & 283,5 & I.74I, 5 & $=$ & - \\
\hline RGM & 16,4 & - & 1.863 & inar. & 2.349 & 445.5 & $2.55 \mathrm{r} .5$ & 一 & $\longrightarrow$ \\
\hline OD & I 1,9 & 445.5 & $\longrightarrow$ & - & 2.187 & 445.5 & $1.822,5$ & 一 & - \\
\hline MOM & 9.8 & 567 & r. 498,5 & arm. & I. $74 x .5$ & 405 & 2.025 & - & - \\
\hline
\end{tabular}


3. |las idmas/

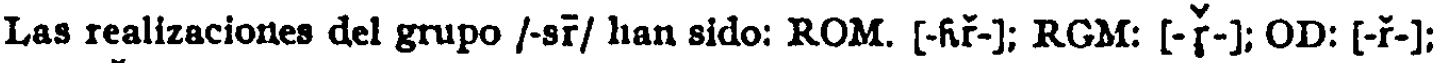
MIOMI: [-š̌-].

\begin{tabular}{|c|c|c|c|c|c|c|c|c|c|}
\hline \multirow{2}{*}{ Inf. } & \multirow[b]{2}{*}{ I } & \multirow[b]{2}{*}{2} & \multirow[b]{2}{*}{3} & \multirow[b]{2}{*}{4} & \multirow[b]{2}{*}{5} & \multicolumn{2}{|c|}{6} & \multicolumn{2}{|c|}{7} \\
\hline & & & & & & $\mathbf{r}_{1}$ & $\mathbf{F}_{2}$ & $T_{1}$ & $T_{2}$ \\
\hline ROM & $I 1,2$ & 324 & $x .984,5$ & arm. & $2.55 \pi .5$ & 729 & I.336,5 & - & $=$ \\
\hline$R G M$ & $\times 3,6$ & 一 & 3.159 & arm. & 3.726 & 972 & x.863 & - & - \\
\hline OD & 11,9 & 465 & 1.377 & Inar. & 2.025 & 567 & I.377 & - & - \\
\hline MOM & 10,5 & 364,5 & $\longrightarrow$ & 一 & 2.794 .5 & 972 & I.74I,5 & - & - \\
\hline
\end{tabular}

\section{4. |isīadl|}

Las realizaciones del grupo /-s $\bar{r}-/$ han sido: ROM [-řr-]; RGM: [- $\breve{r}-]$; OD: $\left[-\check{r}_{-}\right]$; IIOM: $[-\check{r}-]$.

\begin{tabular}{|c|c|c|c|c|c|c|c|c|c|}
\hline \multirow{2}{*}{ Inf. } & \multirow{2}{*}{$\mathbf{I}$} & \multirow{2}{*}{2} & \multirow[b]{2}{*}{3} & \multirow[b]{2}{*}{4} & \multirow[b]{2}{*}{5} & \multicolumn{2}{|c|}{6} & \multicolumn{2}{|c|}{7} \\
\hline & & & & & & $F_{1}$ & $\mathrm{~F}_{2}$ & $\mathrm{~T}_{1}$ & $T_{2}$ \\
\hline ROAY & 13,6 & 405 & 1.600 & inar. & 2.227 .5 & 688,5 & $1.74 x, 5$ & + & - \\
\hline RGM & 14.7 & 507 & 3.240 & iuar. & 3.726 & $93 x, 5$ & $2.146,5$ & - & + \\
\hline OD & II,5 & 364,5 & I.70r & inar. & 2.511 & 931.5 & I. 539 & $=$ & + \\
\hline MOM & 16,8 & - & 一 & - & $2.632,5$ & 1.053 & 2.065 .5 & - & - \\
\hline
\end{tabular}




\section{5. |los ístos|}

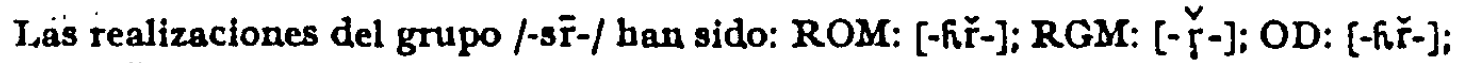
MOM: [-fřr-].

\begin{tabular}{|c|c|c|c|c|c|c|c|c|c|}
\hline Inf. & $\mathrm{I}$ & 2 & 3 & 4 & 5 & $\mathrm{~F}_{1}$ & $\mathrm{~F}_{1}$ & $\mathrm{~T}_{1}$ & $\mathrm{~T}_{1}$ \\
\hline ROM & 9.4 & 364.5 & 1.215 & arm. & I.539 & 486 & 1.134 & - & + \\
\hline RGM & 16.1 & - & 1.741 .5 & Inar. & 2.430 & 567 & 1.377 & - & + \\
\hline OD & 8.4 & 526.5 & 1.255 .5 & arm. & 1.579 .5 & 526.5 & 1.215 & - & - \\
\hline MOM & 13.6 & 475 & 1.458 & arm. & 1.782 & 486 & 1.336 .5 & - & + \\
\hline
\end{tabular}

\section{6. |los romános/}

I as realizaciones del grupo /-s $\bar{r}-/$ han sido: ROM: [-fhř-]; RGM: $[-\check{r}-]$; OD: $[-\check{r}-]$; MOM: $[-\check{r}-]$.

\begin{tabular}{|c|c|c|c|c|c|c|c|c|c|}
\hline Inf. & $I$ & 2 & 3 & 4 & 5 & $F_{1}$ & $F_{1}$ & $T_{1}$ & $T_{2}$ \\
\hline ROM & 7.3 & 445.5 & 1.215 & arm. & 1.539 & 486 & 1.134 & + & + \\
\hline IGM & I3 & - & 2.389 .5 & inar. & 2.632 .5 & 486 & 1.296 & - & + \\
\hline OD & 9.4 & 400 & 1.255 .5 & inar. & 2.106 & 526.5 & 1.174 .5 & - & + \\
\hline MOM & I3.3 & 364.5 & - & - & 1.620 & 445.5 & 1.174 .5 & - & + \\
\hline
\end{tabular}


7. |los irusos|

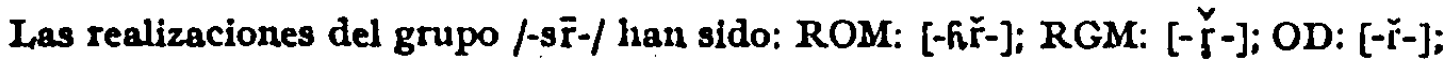
MOM: [-hr̆-].

\begin{tabular}{|c|c|c|c|c|c|c|c|c|c|}
\hline Inf. & 1 & 2 & 3 & 4 & 5 & $F_{2}$ & $F_{2}$ & $T_{1}$ & $T_{2}$ \\
\hline ROM & 7.7 & 526,5 & I.215 & arm. & 1.458 & 324 & 972 & - & + \\
\hline RGM & 14.7 & - & I.579.5 & inar. & 2.551 .5 & 324 & 567 & - & + \\
\hline OD & 9.8 & 567 & I.134 & inar. & 2.187 & 364.5 & 931.5 & - & - \\
\hline MOM & 12.6 & 567 & 1.417 .5 & arm. & 2.511 & 364 & 688.5 & - & - \\
\hline
\end{tabular}

\section{CONCLUSIONES}

I. La duración de [r] en sflaba tónica es de I2,25 cs; en sflaba. átona, $12,36 \mathrm{cs}$, algo mayor, por lo tanto.

2. De los 28 casos analizados, en 7 se ha manifestado la realización sorda (los rizos, los remos, las ramas, los rotos, los rusos, los romanos, de RGM, e Israel, de MOM), y en 2, ensordecida (Israel, de ROM y $R G M)$. En los $2 I$ casos sonoros, el $F_{1}$ ha alcanzado una altura media de 459,I9 $\mathrm{Hz}$.

3. $\mathrm{E} 1 \mathrm{~F}_{2}$ de $[\tilde{\mathrm{r}}]$ no aparece en 4 casos (los remos, de $\mathrm{OD}$, las ramas, los romanos, Israel, de MOM); en los demás alcanza las siguientes frecuencias medias: con $/ \mathrm{i} /: \mathrm{I} .508,62 \mathrm{~Hz}$; con $/ \mathrm{e} /: \mathrm{I} .5 \mathrm{I2} \mathrm{Hz}$; con /a/: 2.I86,9I $\mathrm{Hz}$; con /o/: I.504,28 Hz; con /u/: I. 336,5 Hz.

4. De los 24 casos en los que aparece $F_{2}$, I2 son inarmónicos y I2 armónicos: cuando es inarmónico, la altura es mayor $(1.768,45 \mathrm{~Hz}$ de valor medio) que cuaudo es armónico (valor medio, $1.528,87 \mathrm{~Hz}$ ). Del mismo modo, cuando es inarmónico, la altura a la que aparece el ruido de fricción es mayor (valor medio, 2.467,12 $\mathrm{Hz}$ ) que cuando es armónico (valor medio, $1.957,27 \mathrm{~Hz}$ ).

5. El comienzo de la fricación se produce a las siguientes frecuencias: $\operatorname{con} / \mathrm{i} /:$ 2.055.37 Hz; con /e/: I.944 Hz; con /a/: I.774,25 Hz; con /o/: I.903,5 Hz; con /u/: $2.176,87 \mathrm{~Hz}$.

6. Las trausiciones del $F_{2}$ de las vocales adyacentes se manifiestan del siguiente modo: con /i, e/ son siempre fuertemente negativas (más 


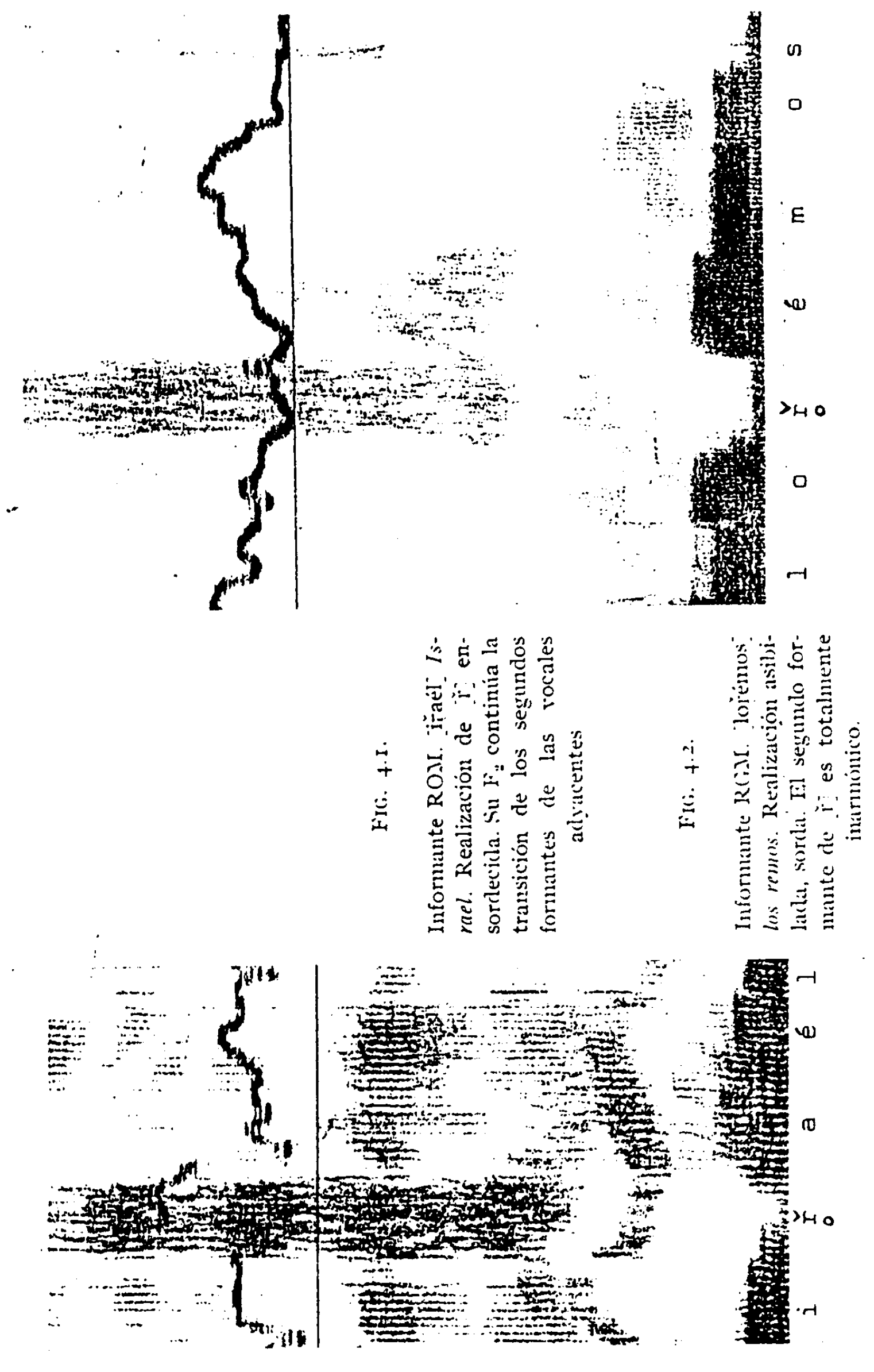



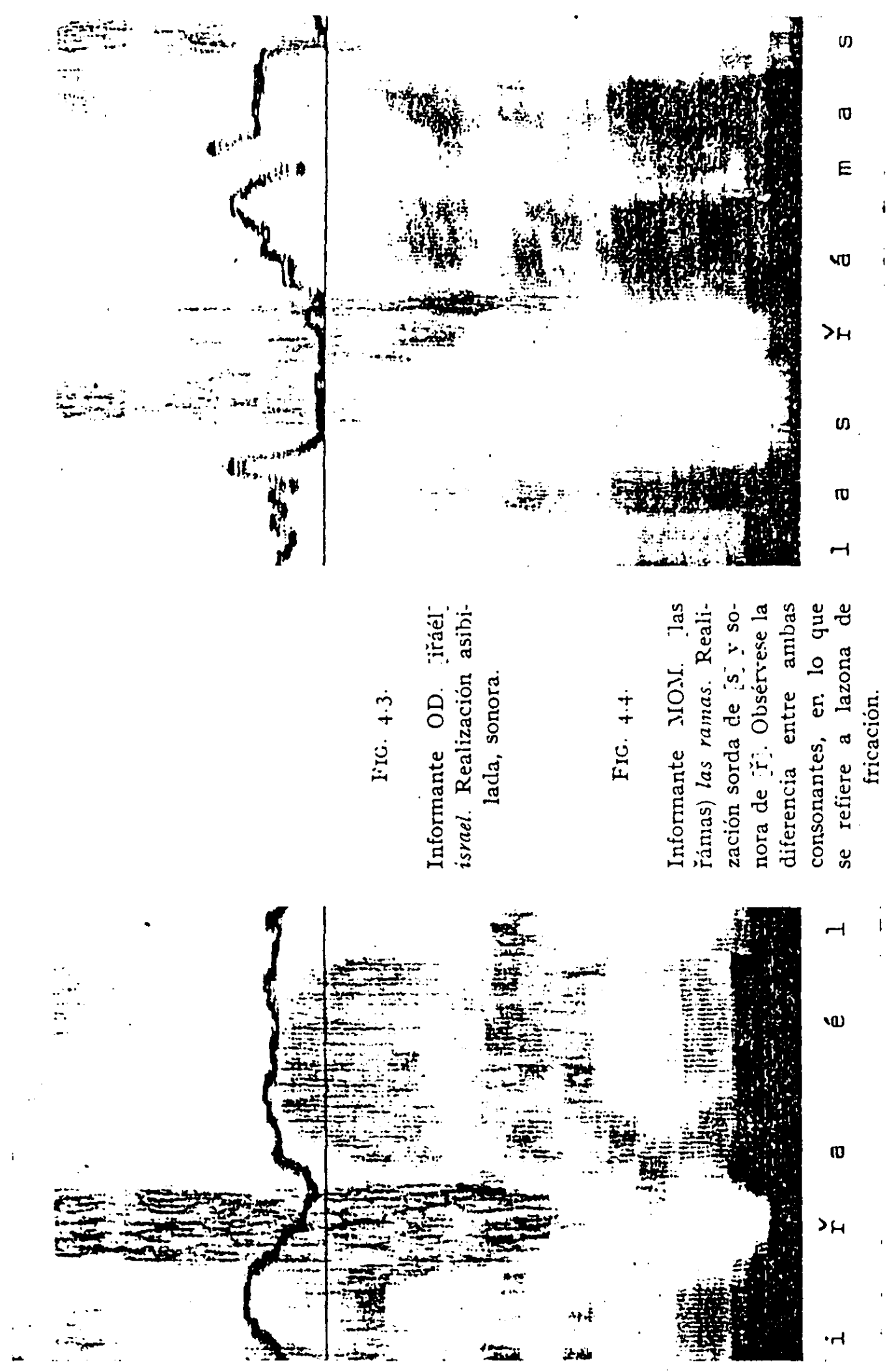
con la primera que con la segunda); con $/ 0 /$ son débilmente positivas, excepto en un caso, en el que aparece como díbilmente negativa; con/u/ son positivas o negativas, independientemente de la relación de frecuencias del $\mathrm{F}_{2}$ de $\left[\mathrm{r}\right.$ ] y del $\mathrm{F}_{2}$ de la vocal siguiente. Con /a/fluctúan entre los tres valores, positivo, horizontal o negativo.

$T_{1}$ aparece negativo en todos los casos, excepto en dos: uno es $(t)$ Israel, de ROM; otro es horizontal: Israel, de OD.

\section{5. [ั] precedido de nasal.}

Hemos analizado nueve casos de secuencias [-nř-]. Los resultados son los siguientes:

I. [un Fio]

\begin{tabular}{|c|c|c|c|c|c|c|c|c|c|}
\hline \multirow{2}{*}{ Inf. } & \multirow{2}{*}{ I } & \multirow{2}{*}{2} & \multirow{2}{*}{3} & \multirow{2}{*}{4} & \multirow{2}{*}{5} & \multicolumn{2}{|c|}{6} & \multicolumn{2}{|c|}{7} \\
\hline & & & & & & $F_{1}$ & $\mathrm{~F}_{2}$ & $T_{1}$ & $T_{2}$ \\
\hline $\mathrm{ROM}$ & 10,1 & 445,5 & $1.660,5$ & arm. & 2.065 .5 & 283.5 & 2.308 .5 & - & - \\
\hline RGM & 14.7 & 324 & I. $74 \mathrm{r} .5$ & inar. & $2.065,5$ & 324 & 3.078 & - & - \\
\hline OD & 7.3 & $5^{26,5}$ & I. 296 & inar. & 2.025 & 364,5 & 2.389 .5 & - & 一 \\
\hline MOM & 16,8 & $5^{67}$ & I.134 & arm. & 2.470 .5 & 364,5 & $2.632,5$ & - & - \\
\hline
\end{tabular}

2. [enrike]

\begin{tabular}{|c|c|c|c|c|c|c|c|c|c|}
\hline \multirow[b]{2}{*}{ Inf. } & \multirow[b]{2}{*}{$\mathbf{I}$} & \multirow[b]{2}{*}{2} & \multirow[b]{2}{*}{3} & \multirow[b]{2}{*}{4} & \multirow[b]{2}{*}{5} & \multicolumn{2}{|c|}{6} & \multicolumn{2}{|c|}{7} \\
\hline & & & & & & $\mathrm{I}_{1}$ & $F_{2}$ & $T_{1}$ & $T_{2}$ \\
\hline ROM & $9, \mathrm{x}$ & 445.5 & I. 498,5 & arm. & 2.106 & 324 & 2.268 & - & - \\
\hline RGMI & 10.5 & 486 & 2.794 .5 & inar. & 2.956 .5 & 243 & 3.159 & - & - \\
\hline OD & 8.7 & 567 & 1.782 & inar. & 2.592 & 445.5 & 2.673 & - & - \\
\hline MOM & 10,5 & $5^{26,5}$ & $x .660,5$ & arun. & 2.430 & 324 & $2.308,5$ & - & - \\
\hline
\end{tabular}


3. [in Flmo]

\begin{tabular}{|c|c|c|c|c|c|c|c|c|c|}
\hline \multirow{2}{*}{ Inf. } & \multirow{2}{*}{$\mathbf{r}$} & \multirow{2}{*}{2} & \multirow{2}{*}{3} & \multirow{2}{*}{4} & \multirow{2}{*}{5} & \multicolumn{2}{|c|}{6} & \multicolumn{2}{|c|}{7} \\
\hline & & & & & & $P_{1}$ & $\mathbf{P}_{\mathbf{8}}$ & $T_{1}$ & $T_{3}$ \\
\hline ROM & $I I, 9$ & 486 & I.377 & arm. & r. $70 \mathrm{r}$ & 445.5 & I.944 & - & - \\
\hline RGM & 22,6 & 526,5 & I. $74^{I}, 5$ & inar. & $2.470,5$ & 405 & 2.835 & - & - \\
\hline OD & 6.3 & 526,5 & $1.336,5$ & arm. & 2.311 & 367 & 2.268 & - & - \\
\hline MOM & 10,8 & 526.5 & 850,5 & arm. & 2.227 .5 & 486 & $1.822,5$ & - & - \\
\hline
\end{tabular}

4. [enrddo]

\begin{tabular}{|c|c|c|c|c|c|c|c|c|c|}
\hline \multirow{2}{*}{ Inf. } & \multirow[b]{2}{*}{ I } & \multirow[b]{2}{*}{2} & \multirow[b]{2}{*}{3} & \multirow[b]{2}{*}{4} & \multirow[b]{2}{*}{5} & \multicolumn{2}{|c|}{6} & \multicolumn{2}{|c|}{7} \\
\hline & & & & & & $F_{1}$ & $I_{2}$ & $T_{1}$ & $T_{2}$ \\
\hline ROM & II,9 & 445.5 & $1.45^{8}$ & arm. & 2.227 .5 & 405 & I. 903.5 & - & - \\
\hline RGMI & 11,9 & $4^{86}$ & 2.349 & inar. & 2.673 & .445 .5 & 2.592 & - & 一 \\
\hline OD & 10,8 & 526,5 & 729 & arm. & $2.470,5$ & 526,5 & $2.389,5$ & - & - \\
\hline MIOMI & $x 0,5$ & 486 & r. 741,5 & aru. & 2.106 & 526,5 & I. 863 & - & - \\
\hline
\end{tabular}




\section{5. [ún Fämo]}

\begin{tabular}{|c|c|c|c|c|c|c|c|c|c|}
\hline \multirow{2}{*}{ Inf. } & \multirow{2}{*}{ I } & \multirow{2}{*}{2} & \multirow{2}{*}{3} & \multirow{2}{*}{4} & \multirow{2}{*}{5} & \multicolumn{2}{|c|}{6} & \multicolumn{2}{|c|}{7} \\
\hline & & & & & & $F_{1}$ & $F_{2}$ & $T_{1}$ & $T_{2}$ \\
\hline ROM & I $x, 9$ & 405 & 1.215 & arw. & 1.620 & 688,5 & $1.4 \simeq 7,5$ & - & - \\
\hline $\mathbf{R G M}$ & 12,6 & 405 & $\mathrm{r} .660,5$ & inar. & 2.349 & $93 r, 5$ & I.74I, 5 & - & + \\
\hline OD & 6,6 & 486 & I.377 & inar. & 2.511 & $93 I, 5$ & I. 498,5 & - & + \\
\hline MOM & I $x, 9$ & 526.5 & 810 & arm. & $1.74 \pi, 5$ & 931,5 & $1.660,5$ & - & - \\
\hline
\end{tabular}

6. [onfrár]

\begin{tabular}{|c|c|c|c|c|c|c|c|c|c|}
\hline \multirow{2}{*}{ I nf. } & \multirow{2}{*}{ I } & \multirow{2}{*}{2} & \multirow{2}{*}{3} & \multirow{2}{*}{4} & \multirow{2}{*}{5} & \multicolumn{2}{|c|}{6} & \multicolumn{2}{|c|}{7} \\
\hline & & & & & & $F_{1}$ & $F_{2}$ & $T_{1}$ & $T_{2}$ \\
\hline $120 M$ & 13.3 & 405 & I. $45^{8}$ & arm. & 1.782 & 688,5 & $1.4 \times 7.5$ & - & - \\
\hline$R G M$ & 14 & $4^{86}$ & r. 660,5 & inar. & $2.308,5$ & 810 & I. 782 & - & + \\
\hline OD & 7.7 & 526,5 & I. 336,5 & arm. & x.944 & $89 \mathrm{r}$ & $1.45^{8}$ & - & + \\
\hline MOM & $I I, 2$ & $5^{26}$ & I. $74 \mathrm{I}, 5$ & arm. & 1.903 .5 & $97^{2}$ & I. $74 \mathrm{r} .5$ & - & - \\
\hline
\end{tabular}




\section{7. [on*a]}

\begin{tabular}{|c|c|c|c|c|c|c|c|c|c|}
\hline \multirow{2}{*}{ Iuf. } & \multirow{2}{*}{$\mathbf{I}$} & \multirow{2}{*}{2} & \multirow{2}{*}{3} & \multirow{2}{*}{4} & \multirow{2}{*}{5} & \multicolumn{2}{|c|}{6} & \multicolumn{2}{|c|}{7} \\
\hline & & & & & & $F_{1}$ & $\mathrm{~F}_{2}$ & $T_{1}$ & $T$, \\
\hline ROM & 9,8 & 405 & 1.377 & inar. & 1.620 & 445.5 & X.215 & - & + \\
\hline RGM & 9.7 & - & I. 660,5 & inar. & 2.592 & 810 & 1.215 & $=$ & $=$ \\
\hline OD & 7.7 & 526,5 & $x .417 .5$ & Inar. & $x .863$ & 769.5 & $1.336,5$ & - & + \\
\hline MOM & $9, x$ & 364,5 & - & - & $2.794,5$ & 931.5 & $\mathbf{1 . 7 4 1 . 5}$ & - & + \\
\hline
\end{tabular}

8. [ún iólo]

\begin{tabular}{|c|c|c|c|c|c|c|c|c|c|}
\hline Inf. & I & 2 & 3 & 4 & 5 & $I_{1}^{2}$ & $F_{2}$ & $T_{1}$ & $T_{2}$ \\
\hline ROMI & 8,7 & 445,5 & 1.215 & arm. & $1.498,5$ & 445.5 & 1.053 & - & + \\
\hline RGM & I0,1 & 364,5 & $1.498,5$ & arm. & $2.308,5$ & 567 & 1.215 & - & $\ddots$ \\
\hline OD & 5.2 & 486 & 1.053 & inar. & 2.470 .5 & 324 & 1.093 .5 & - & - \\
\hline MOMI & 8.4 & 486 & 1.336 & arm. & 1.620 & 486 & 1.255 .5 & - & $\ddots$ \\
\hline
\end{tabular}




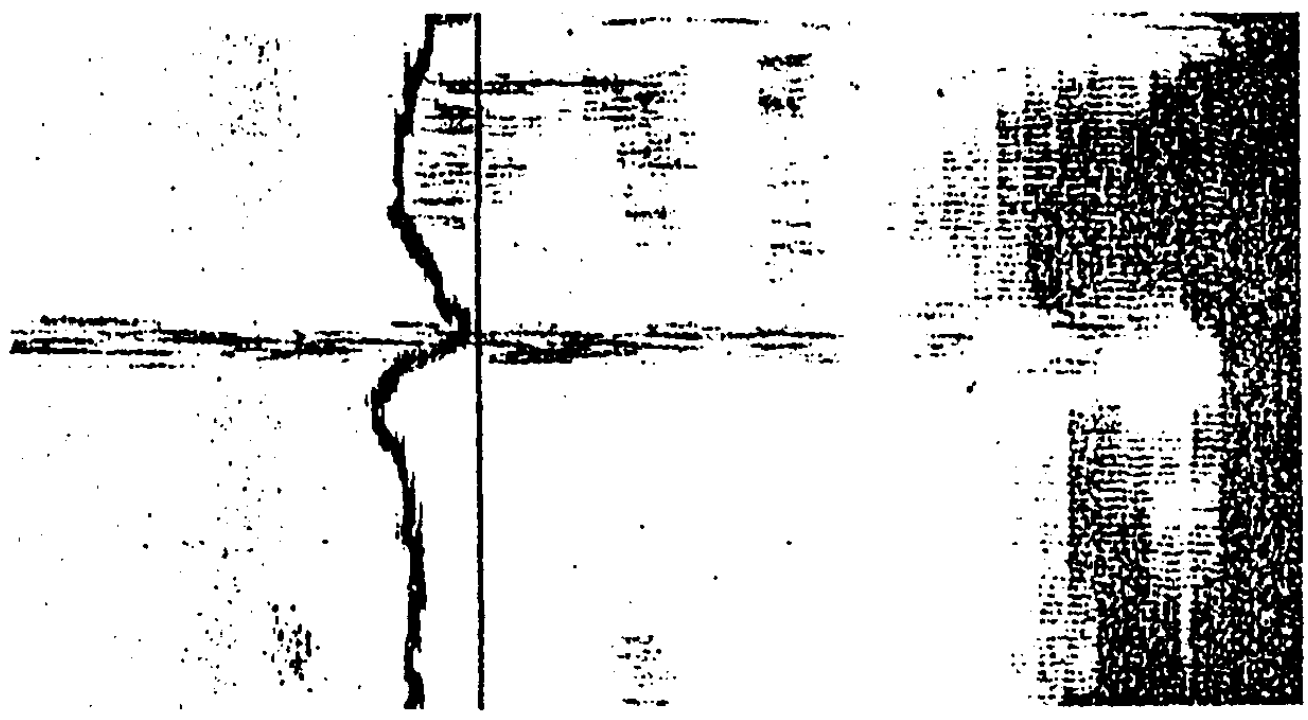

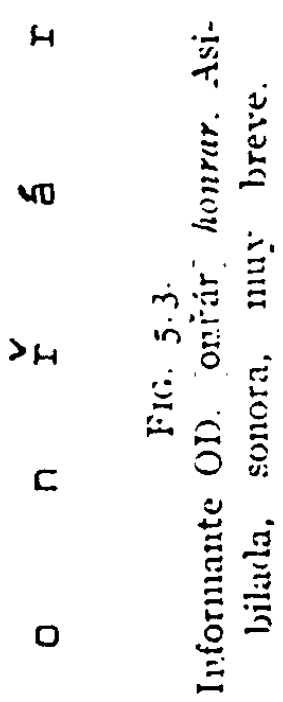
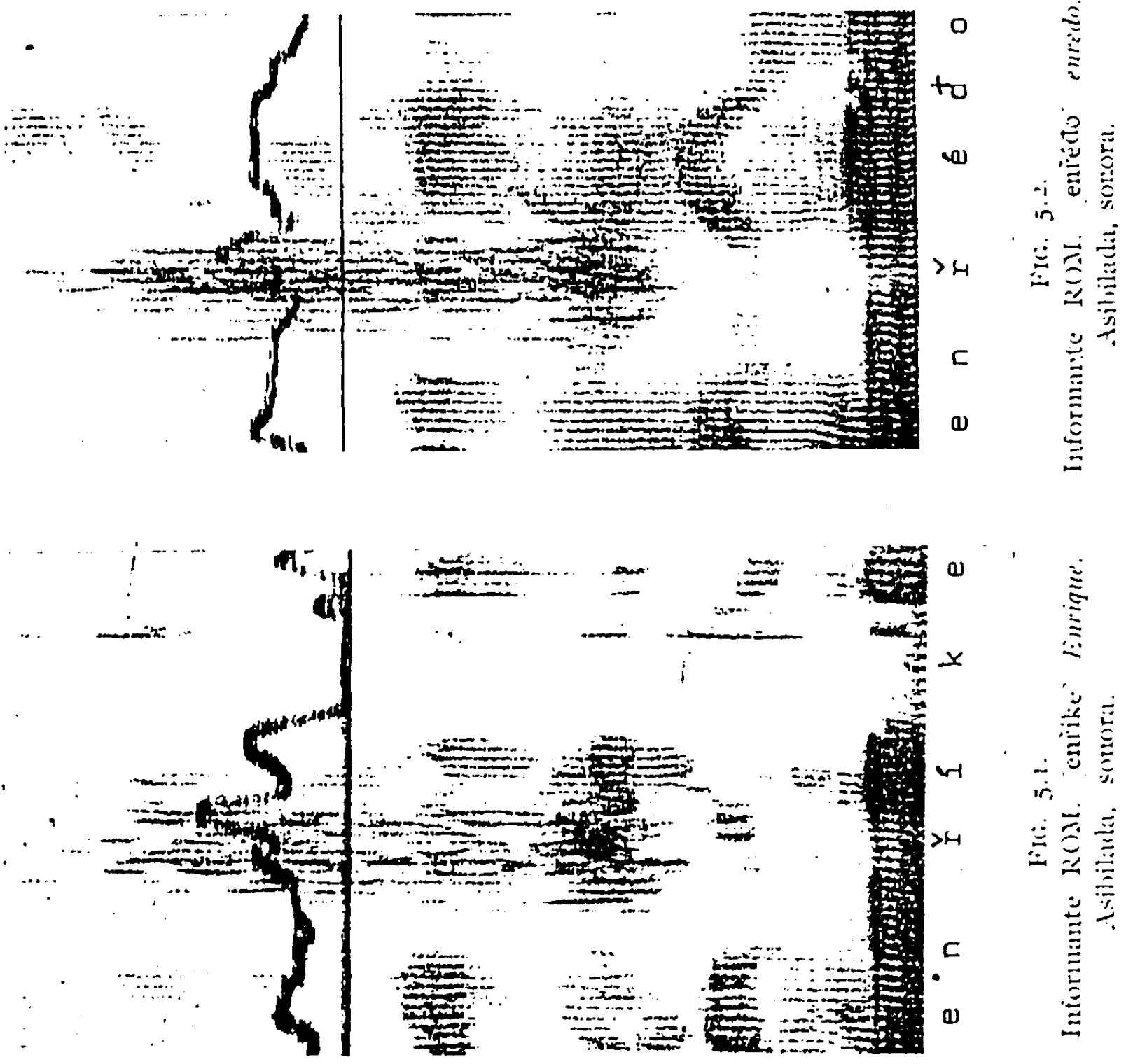


9. [in Fiso]

\begin{tabular}{|c|c|c|c|c|c|c|c|c|c|}
\hline \multirow{2}{*}{ Inf. } & \multirow[b]{2}{*}{ I } & \multirow[b]{2}{*}{2} & \multirow[b]{2}{*}{3} & \multirow[b]{2}{*}{4} & \multirow[b]{2}{*}{5} & \multicolumn{2}{|c|}{6} & \multicolumn{2}{|c|}{7} \\
\hline & & & & & & $F_{1}$ & $P_{2}$ & $T_{1}$ & $T_{2}$ \\
\hline ROM & 9,4 & 486 & 1.093 .5 & arm. & 1.377 & 283.5 & 850,5 & - & + \\
\hline RGM & 12,6 & 364,5 & $x .498,5$ & inar. & 2.065 .5 & 283.5 & $x .053$ & - & + \\
\hline OD & 6,6 & 445.5 & I.174.5 & inar. & 2.754 & 324 & $\mathrm{r} .053$ & - & + \\
\hline MOM & II, 2 & $.526,5$ & 810 & arm. & 2.187 & 445.5 & 688,5 & - & + \\
\hline
\end{tabular}

\section{CONCLUSIONES}

De los cuadros anteriores, podemos obtener las siguientes conclusiones:

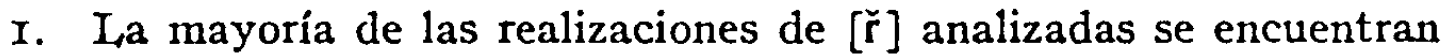
en sllaba tónica; su duración en ella es de 10,49 cs, mientras que en silaba átona es de $9.7 \mathrm{cs}$.

2. De los 36 casos analizados, tres se presentan ensordecidos en su parte final: Enrique, enredo, honrar, de RGM, y uno sordo: honra, del mismo informante. El valor medio del límite superior de $F_{1}$ es de 473,25 $\mathrm{Hz}$.

3. $\mathrm{F}_{2}$ de $[\check{\mathrm{r}}]$ alcanza los siguientes valores: con $/ \mathrm{i} /: \mathrm{r} \cdot 696,3 \mathrm{I} \mathrm{Hz}$ con /e/: I.447,87 Hz; con /a/: I.446,17 Hz; con /o/: $1.275,62 \mathrm{~Hz}$; con /u/: $\mathrm{x} .444,37 \mathrm{~Hz}$.

4. Este $F_{2}$ se manifiesta como armónico en 20 casos, y como inarmónico en I5. En un caso no aparece.

Cuando es armónico, su frecuencia es más baja (valor medio, r.298 $\mathrm{Hz}$ ), mientras que inarmónico es más alta (valor medio, I.638,9 Hz).

Cuando $F_{2}$ de [r] es armónico, la frecuencia a la que aparece la fricación del mencionado alófono es menor (valor medio, $2.014,87 \mathrm{~Hz}$ ) que cuando es inarmónico (valor medio, 2.354,36 Hz).

5. Iil comienzo de la fricación se produce a las siguientes frecuencias: con /i/: 2.338,87 Hz; con /e/: 2.298,37 Hz; con /a/: $2.085 .75 \mathrm{~Hz}$; con /o/: $\mathrm{r} .974,37 \mathrm{~Hz}$; con /u/: $2.095,87 \mathrm{~Hz}$. 
6. Las transiciones de la vocal siguiente a [ř] aparecen con lós siguientes movimientos:

$T_{2}$ es muy negativa con $/ i$, e/. Es siempre positiva con $/ u /$ Con $/ a /$ varia, sin que observemos relación con la altura del $F_{2}$ de [ř $]$. En el informante MOM, en el que $T_{2}$ es en este caso $(-)$, aparece $(+)$ en honra, donde no existe $\mathrm{F}_{2}$. De los 12 casos, siete son positivos. Además, es menester señalar que todas estas transiciones son muy poco marcadas, aunque generalmente largas. Con /o/ las transiciones son también muy pequeñas y oscilan entre $(+)$ y $(-)$.

$\mathrm{T}_{1}$ es siempre negativa.

\section{6. [†] precedido de lateral.}

En este grupo hemos analizado los alófonos [r] precedidos de conlsonante $/ 1 /$. cuyos valores damos a continuación:

I. [el Fío]

\begin{tabular}{|c|c|c|c|c|c|c|c|c|c|}
\hline \multirow{2}{*}{ Inf. } & \multirow[b]{2}{*}{$\mathbf{I}$} & \multirow[b]{2}{*}{2} & \multirow[b]{2}{*}{3} & \multirow[b]{2}{*}{4} & \multirow[b]{2}{*}{5} & \multicolumn{2}{|c|}{6} & \multicolumn{2}{|c|}{7} \\
\hline & & & & & & $\mathrm{I}_{1}$ & $\mathbf{F}_{2}$ & $T_{1}$ & $T_{8}$ \\
\hline ROM & 13 & 486 & $1.498,5$ & arw. & 1.863 & 324 & $2.3^{8} 9.5$ & - & $\longrightarrow$ \\
\hline RGM & 14 & 364,5 & 2.187 & inar. & 2.835 & 283,5 & $3 . \times 99.5$ & - & - \\
\hline OD & 10 & 486 & 1.620 & inar. & 2.713 .5 & 329 & $2.794,5$ & - & - \\
\hline MOM & 13,6 & 526,5 & I. $45^{8}$ & arm. & 2.106 & 364,5 & 2.592 & - & - \\
\hline
\end{tabular}




\section{2. [el Fél]}

\begin{tabular}{|c|c|c|c|c|c|c|c|c|c|}
\hline Inf. & $I$ & 2 & 3 & 4 & 5 & $F_{1}$ & $F_{2}$ & $T_{1}$ & $T_{2}$ \\
\hline ROM & 11,2 & 405 & 1.553 & arm. & 2.025 & 445.5 & r.741.5 & - & - \\
\hline RGM & 15.7 & 364.5 & 2.227 .5 & inar. & 2.754 & 526.5 & 2.835 & - & - \\
\hline OD & 8.7 & 486 & 1.458 & Inar. & 2.794 .5 & 405 & 2.227 .5 & - & - \\
\hline MOM & 13.3 & 567 & 1.579 .5 & arm. & 2.106 & 486 & 2.065 .5 & - & - \\
\hline
\end{tabular}

3. [alfededor]

\begin{tabular}{|c|c|c|c|c|c|c|c|c|c|}
\hline \multirow{2}{*}{ Inf. } & \multirow{2}{*}{ I } & \multirow{2}{*}{2} & \multirow{2}{*}{3} & \multirow{2}{*}{4} & \multirow{2}{*}{5} & \multicolumn{2}{|c|}{6} & \multicolumn{2}{|c|}{7} \\
\hline & & & & & & $F_{1}$ & $F_{2}$ & $T_{1}$ & $T_{2}$ \\
\hline ROM & 9,8 & 445,5 & I. 498,5 & arm. & 2.025 & 324 & 1.782 & 一 & - \\
\hline $\mathrm{RGM}$ & $r 0,5$ & 324 & 2.227 .5 & inar. & 2.916 & 445.5 & 2.349 & - & 一 \\
\hline OD & 8 & 324 & - & - & $2.632,5$ & 405 & 2.106 & - & - \\
\hline MOM & 7.7 & 567 & $\mathbf{x . 0 9 3 . 5}$ & arm. & $2.146,5$ & 405 & I. 782 & 一 & - \\
\hline
\end{tabular}


4. [cl fdyo]

\begin{tabular}{|c|c|c|c|c|c|c|c|c|c|}
\hline \multirow{2}{*}{ Inf. } & \multirow{2}{*}{$x$} & \multirow{2}{*}{2} & \multirow{2}{*}{3} & \multirow{2}{*}{4} & \multirow{2}{*}{5} & \multicolumn{2}{|c|}{6} & \multicolumn{2}{|c|}{7} \\
\hline & & & & & & $i_{1}$ & $I_{3}$ & $T_{1}$ & $T_{2}$ \\
\hline ROM & $I I, 2$ & 445.5 & $x .45^{8}$ & inar. & $\mathbf{I . 8 2 2 , 5}$ & 729 & 1.539 & 一 & 一 \\
\hline RGM & I5 & 486 & 2.146 & inar. & 2.673 & 972 & r. 944 & - & + \\
\hline OD & 10,1 & 405 & $x .45^{8}$ & inar. & 2.754 & $89 x$ & $x .45^{8}$ & - & + \\
\hline MOM & 12,2 & 567 & 891 & arm. & 2.146 .5 & $1.012,5$ & I. $74 x, 5$ & - & - \\
\hline
\end{tabular}

5. [roto]

\begin{tabular}{|c|c|c|c|c|c|c|c|c|c|}
\hline \multirow{2}{*}{ Iuf. } & \multirow{2}{*}{ I } & \multirow{2}{*}{2} & \multirow{2}{*}{3} & \multirow{2}{*}{4} & \multirow{2}{*}{5} & \multicolumn{2}{|c|}{6} & \multicolumn{2}{|c|}{7} \\
\hline & & & & & & $F_{1}$ & $F_{2}$ & $T_{1}$ & $T_{2}$ \\
\hline ROM & $I I, 2$ & 445,5 & 1.296 & ann. & I. 579.5 & 445.5 & 972 & 一 & 一 \\
\hline RGM & 13.3 & 364,5 & I. 579.5 & inar. & 2.430 & 567 & I. 74,5 & 一 & - \\
\hline OD & 8 & $4^{86}$ & 一 & - & 2.592 & 405 & I.I 74,5 & 一 & - \\
\hline MIOM & 7.3 & 567 & I. $45^{8}$ & arm. & 2.430 & 405 & I.296 & 一 & - \\
\hline
\end{tabular}



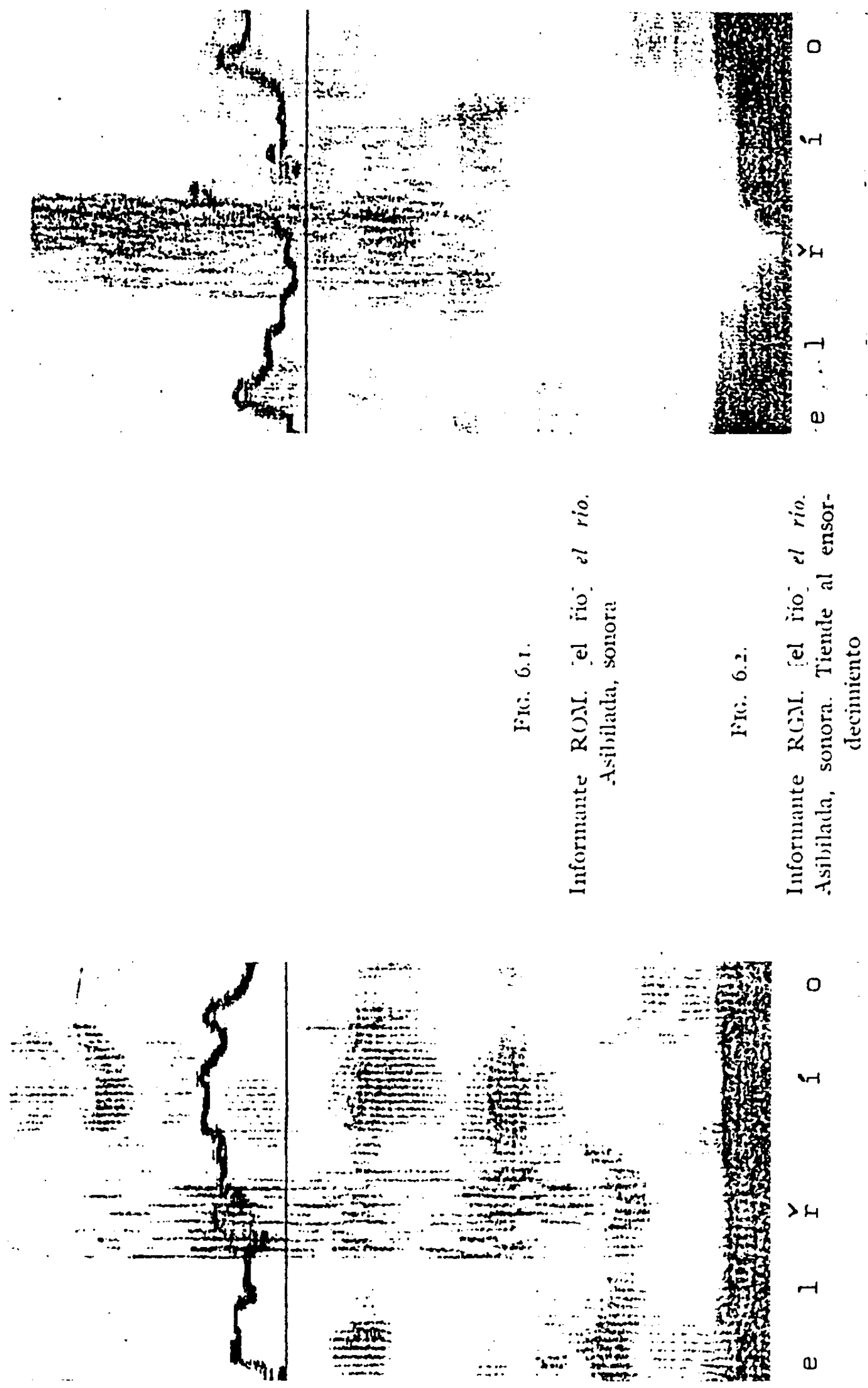

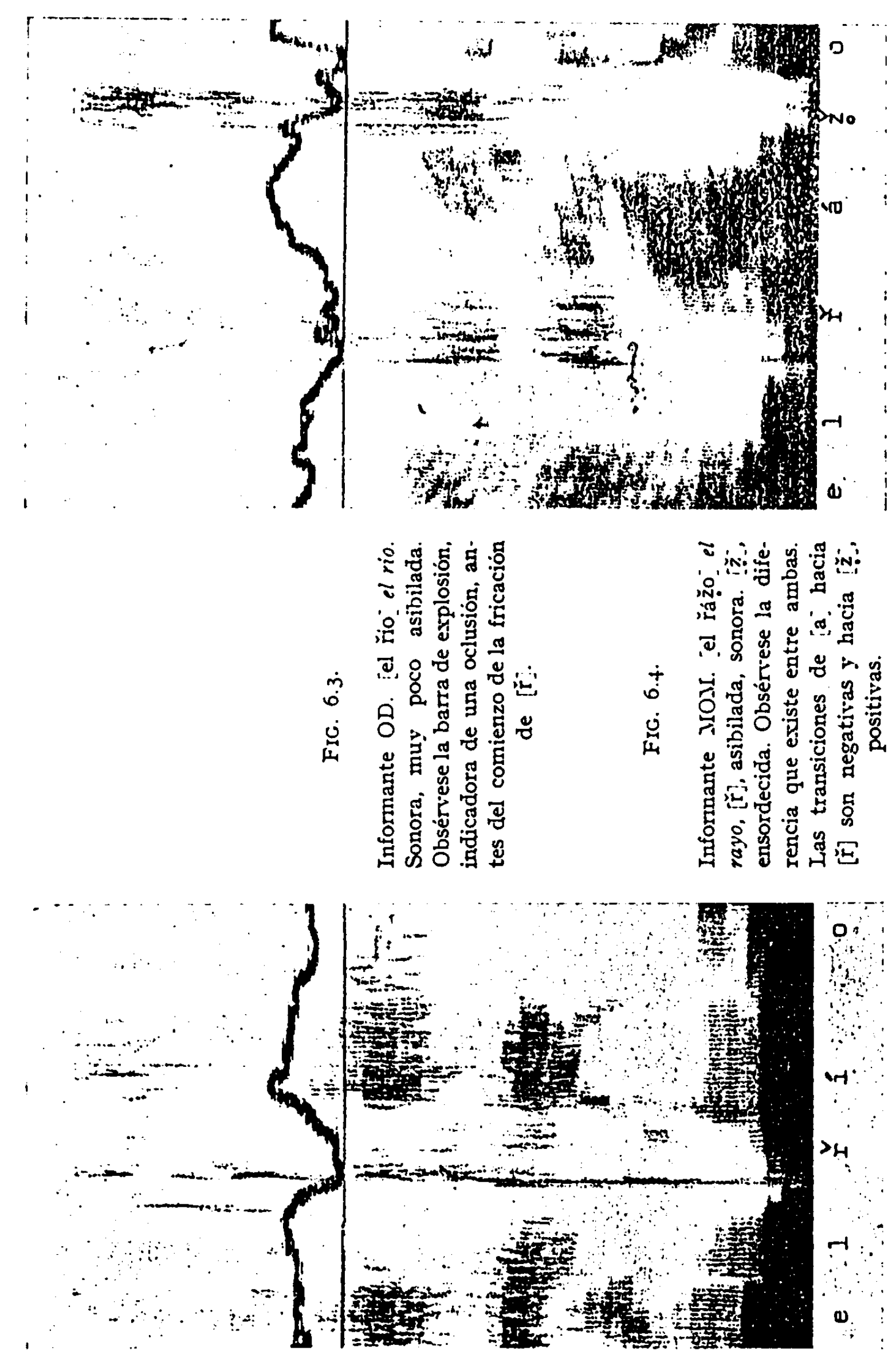
6. [el r̀iso]

\begin{tabular}{|c|c|c|c|c|c|c|c|c|c|}
\hline Inf. & 1 & 2 & 3 & 4 & 5 & $F_{1}$ & $F_{2}$ & $T_{1}$ & $T_{2}$ \\
\hline IROM & 8 & 445.5 & 1.296 & inar. & 1.539 & 324 & 972 & - & + \\
\hline RGM & 12,9 & 364,5 & 2.025 & inar. & 2.673 & 283.5 & 1.47 .5 & - & + \\
\hline OD & 7 & 486 & I.134 & inar. & 2.268 & 324 & 972 & - & + \\
\hline $\mathrm{MOOM}$ & 8,4 & 567 & $\mathrm{I.458}$ & inar. & $2.470,5$ & 526 & 1.296 & - & + \\
\hline
\end{tabular}

\section{CONCLUSIONES}

De los cuadros expuestos podemos deducir las siguientes conclusiones:

I. La duración media de [ř en sílaba tónica - la mayoría de los casos- es de II,2 cs, mientras que en sílaba átona es de 9 cs.

2. In los 24 casos analizados en este apartado, han aparecido dos ensordecidos en su parte final, pertenecientes al informante RGM: el rayo, el roto. La altura del $\mathrm{F}_{1}$ alcanza un valor medio de $457,3 \mathrm{I} \mathrm{Hz}$.

3. Los valores medios del $\mathrm{F}_{2}$ de [r] ] son los siguientes: con /i/: I.690,87

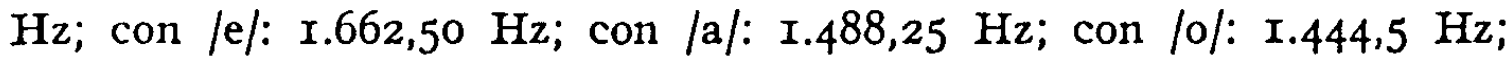
$\operatorname{con} / \mathrm{u} /: \mathrm{I} .478,25 \mathrm{~Hz}$.

4. Este $\mathrm{F}_{2}$ de $[\check{\mathrm{r}}]$ ha aparecido con una conformación plenamente armónica en nueve casos, e inarmónica, en 13.

Cuando es armónico, su frecuencia es menos alta (valor medio, I.369.55 Hz) que cuando es inarmónico (valor medio, I.7I0,34 Hz) Del mismo modo, cuando es armónico, la turbulencia de la fricción aparece a un nivel más bajo (valor medio, 2.053,05 Hz) que cuando es inarmónico (valor medio, $2.5 \mathrm{II} \mathrm{Hz}$ ).

5. Iil comienzo de la fricación del $\mathrm{F}_{2}$ de $[\check{\mathrm{r}}]$ se realiza a las siguientes frecuencias: con /i/: 2.379,37 Hz; con /e/: $2.424,93 \mathrm{~Hz}$; con /a/: 2.349 $\mathrm{Hz}$; con $/ \mathrm{o} /: 2.257,87 \mathrm{~Hz}$; con $/ \mathrm{u} /: 2.237,62 \mathrm{~Hz}$.

6 . Las transiciones de las vocales siguientes a $[\check{r}]$ dan los siguientes movimientos:

$\mathrm{T}_{2}$ es fuertemente negativa con $/ \mathrm{i}, \mathrm{e} /$. Es positiva con $/ \mathrm{u} /$. Con $/ \mathrm{o} /$ es positiva en todos los casos, menos en uno, que se manifiesta como negativa: el roto, de MOM. Con /a/ es débilmente positiva (dos casos) - negativa (dos casos). 


\section{Diferencias entre [r] $y[\mathbf{s}]$.}

Al realizarse $|\bar{r}|$ como [ $[\mathbf{r}]$, adquiere una configuración acústica que lo encuadra en el grupo de los alófonos fricativos de carácter sibilante. A pesar de su semejanza, existen diferencias auditivas, cuyo origen acústico vamos a tratar de establecer aquil.

I. Cuando la asibilada es sonora, mantiene un primer formante de tal anchura en gama de frecuencias, que la distingue netamente de [s]. Si, además, aparece el $\mathrm{F}_{2}$ armónico, la diferencia aumenta.

2. Aun en los casos en que se realiza sin sonoridad o parcialmente ensordecida, la frecuencia del comienzo de la fricación es muy diferente en ambos sonidos. Veamos estos valores medios en cada informante:

\begin{tabular}{|c|c|c|}
\hline Inf. & {$[\mathrm{s}]$} & {$[\check{\text { r̆}]}$} \\
\hline ROM & $2.600 \mathrm{~Hz}$ & $\mathbf{1 . 7 3 0 , 2 6 \mathrm { Hz }}$ \\
\hline RGM & $4.110,15 \mathrm{~Hz}$ & $2.733 \mathrm{~Hz}$ \\
\hline OD & $3.578,25 \mathrm{~Hz}$ & $2.097,21 \mathrm{~Hz}$ \\
\hline NOM & $4.215 \mathrm{~Hz}$ & $2.103 .28 \mathrm{~Hz}$ \\
\hline
\end{tabular}

La mayor frecuencia de la fricación en la realización de [s] se ex-

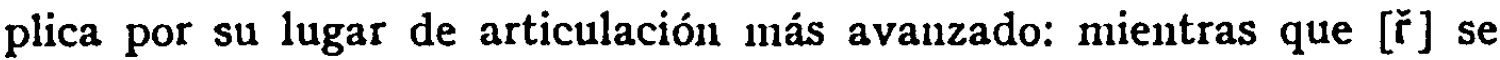
mantiene en todos los informantes como alveolar, [s] oscila entre predorsodentoalveolar y dental, con la consiguiente reducción del resonador anterior y el aumento de su gama de frecuencias.

3. Otro dato también interesante es el límite máximo de frecuencias alcanzado por cada uno de estos sonidos:

\begin{tabular}{|c|c|c|}
\hline Inf. & [s] M11́s de: & [ř hasta: \\
\hline ROM & $25.000 \mathrm{~Hz}$ & $12.800 \mathrm{~Hz}$ \\
\hline RGM & $16.000 \mathrm{~Hz}$ & $13.550 \mathrm{~Hz}$ \\
\hline OD & $16.000 \mathrm{~Hz}$ & $10.348 \mathrm{~Hz}$ \\
\hline MOM & $16.000 \mathrm{~Hz}$ & $10.361 .5 \mathrm{~Hz}$ \\
\hline
\end{tabular}


TYPE B SÖNAGRAM @ KAY ELECTRIC CO. PINE BROOK, N. J.

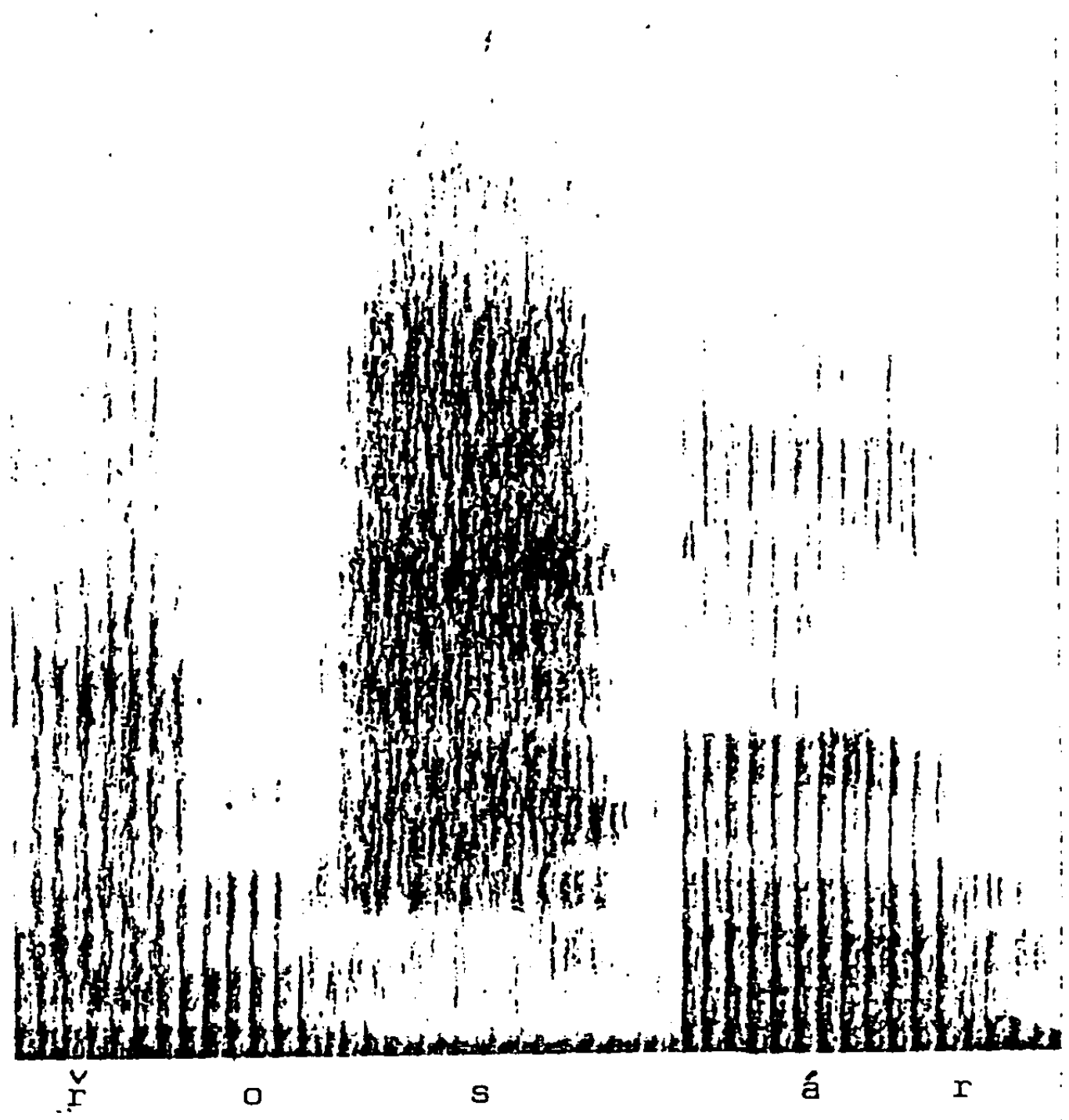

lili: 7.1 .

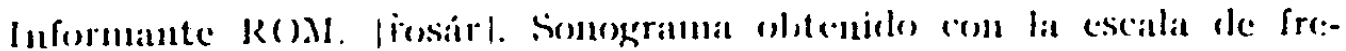
cuencias comprendida contre lfoo y 16.000 11\% para comprobar la altura alcanzarlat por iri? y s s. 
Al alcanzar [s] unas frecuencias altas muy superiores a [r] le imprime un carácter más agudo, carácter que se ve incrementado por su comienzo más alto.

4. Las transiciones de los segundos formantes también varian:

a) en $[\mathrm{s}+\mathrm{i}], \mathrm{T}_{2}$ es horizontal, mientras que con la asibilada es fuertemente negativa;

b) en [s $+\mathrm{e}$ ], $\mathrm{T}_{2}$ es positiva con ROM y RGM, horizontal o nuy poco negativa en $O D$ y alternando entre muy poco positiva o muy poco negativa en MOM, mientras que con [ĩ ] es constantemente negativa.

c) en $[s+a], T_{2}$ es constantemente muy positiva, mientras que con [r] alternan los tres valores, y no muy acentuados.

d) en $[s+o], T_{2}$ es positiva en todos los casos menos en ROM, que es horizontal. Con [r] predomina la transición positiva, aunque aparecen los otros dos valores.

e) en $[\mathrm{s}+\mathrm{u}], \mathrm{T}_{2}$ es positiva en todos los informantes menos en OD, que es horizontal. Con [r] ], predomina la transición positiva, aunque aparecen los otros dos valores.

5. Tanto en [ř como en [s] (y también en [ž, ] como veremos más adelante) aparecen concentraciones de energía en su espectro. Hemos observado que estas concentraciones están situadas a distintas frecuencias, y tienen distintas alturas. Su somienzo (c) y su final (f), para cada informante, dan los siguientes valores:

a) en ROM:

\begin{tabular}{|c|c|c|}
\hline & {$[\check{i}]$} & {$[s]$} \\
\hline$c$ & $1.87^{2}, 7^{2} \mathrm{~Hz}$ & $3.826,66 \mathrm{~Hz}$ \\
\hline $\mathrm{f}$ & $6.749,09 \mathrm{~Hz}$ & $11.426,66 \mathrm{~Hz}$ \\
\hline
\end{tabular}

b) en R(iM:

\begin{tabular}{|c|c|c|}
\hline & {$[\check{r}]$} & {$[s]$} \\
\hline$c$ & $3.317 .33 \mathrm{~Hz}$ & $5.946,66 \mathrm{~Hz}$ \\
\hline$f$ & $9.930 .66 \mathrm{~Hz}$ & $12.485 .33 \mathrm{~Hz}$ \\
\hline
\end{tabular}


c) en OD:

\begin{tabular}{|c|c|c|}
\hline & {$[\check{r}]$} & {$[s]$} \\
\hline$c$ & $2.673 .33 \mathrm{~Hz}$ & $4.293 .33 \mathrm{~Hz}$ \\
\hline$f$ & $6.312,88 \mathrm{~Hz}$ & $12.624 \mathrm{~Hz}$ \\
\hline
\end{tabular}

d) en MOM:

\begin{tabular}{|c|c|c|}
\hline & {$[\check{r}]$} & {$[8]$} \\
\hline$c$ & $1.130,66 \mathrm{~Hz}$ & $5.706,66 \mathrm{~Hz}$ \\
\hline$f$ & $0.947 .33 \mathrm{~Hz}$ & $12.240 \mathrm{~Hz}$ \\
\hline
\end{tabular}

Il comienzo de la concentración de [̌̆] en cada informante es menor (valor medio, 2.248,5 Hz) que el de [s] (valor medio, 4.943.33 Hz),

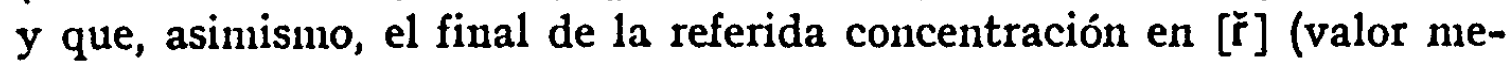
dio, 7.485 Hz) es menor que en [s] (valor medio, I2.194 Hz). De los diferentes valores de comienzo y fin de esta zona de energía, se deduce fácilmente que su anchura es también diferente para [r̆] (valor medio, $5.150,13 \mathrm{~Hz}$ ) y para [s] (valor medio, 7.283,85 Hz).

6. El grado de intensidad de [s] es mayor que el de [ř $]$.

\section{Diferencias entre $[\check{r}] y[z]$.}

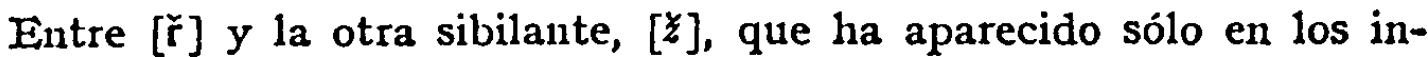
formantes RGM y MOM existen las siguientes diferencias:

I. Cuaudo [z] es plenamente sonoro, el formante bajo es mucho

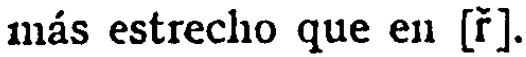

2. Los comienzos de las fricaciones se producen a distinta altura. Ia media de comienzo en RGM se establece en los $2.900 \mathrm{~Hz}$; supera ell un caso los $16.000 \mathrm{~Hz}$; en otro alcanza hasta los $\mathrm{Ir} .360 \mathrm{~Hz}$ (donde [r] llegaba hasta los $9.920 \mathrm{~Hz}$ y [s] superaba los $16.000 \mathrm{~Hz}$ ).

In MOMI, el comienzo de la fricación de [₹] se produce en los $2.831,25$ $\mathrm{Hz}$ y llega hasta los II.440. 
3. El comienzo y el final de la zona donde se encuentra concentrada la energla de su espectro da los siguientes valores medios para [z]:

\begin{tabular}{|c|c|c|}
\hline & $\mathrm{RGM}$ & MOM \\
\hline $\mathrm{c}$ & $3.866,66 \mathrm{~Hz}$ & $3.687 .50 \mathrm{~Hz}$ \\
\hline $\mathrm{f}$ & $9.653 .33 \mathrm{~Hz}$ & $9.840 \mathrm{~Hz}$ \\
\hline
\end{tabular}

Ocupa, como vemos, una posición intermedia entre $[\check{\mathbf{r}}]$ y $[\mathrm{s}]$.

4. La duración de la constricción de [z] es mayor que la de [r]].

5. Las transiciones de los segundos formantes en los casos analizados son positivas con todas las vocales en ambos informantes,

\section{Caracterización individual.}

Los rasgos que caracterizan individualmente a cada uno de los informantes, son los siguientes:

I. ROM: De las 60 realizaciones de [r̆ $]$, dos están ensordecidas:

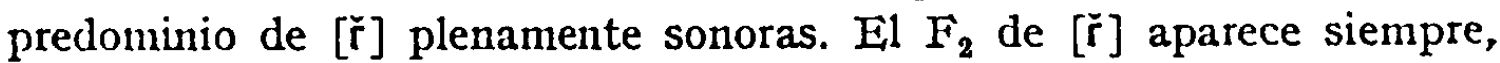
y predomina su realización armónica (42 casos). La fricación de [ŕ $]$ comienza a una frecuencia media de $\mathrm{I} \cdot 730,26 \mathrm{~Hz}$. Auditivamente, se percibe como una consonante muy asibilada y muy sonora.

2. RGM: Han aparecido I2 realizaciones sordas y 22 ensordecidas. $\mathrm{E}_{1} \mathrm{~F}_{2}$ de [r̆] no aparece en dos casos. Este $\mathrm{F}_{2}$ es armónico sólo en ocho realizaciones e inarmónico, en 50 . La fricación de [ř comienza a una frecuencia media de $2.733 \mathrm{~Hz}$. Auditivamente se percibe como una consonante muy asibilada, pero menos sonora que en el informante anterior.

3. $O D$ : Presenta cuatro realizaciones ensordecidas. $E_{1} F_{2}$ de [ř $]$ no aparece en cinco casos; es armónico en 22 ocurrencias, e inarmónico en 33. La fricación de [ř ] comienza a una frecuencia media de $2.097,21 \mathrm{~Hz}$. En este informante, [ $\check{r}$ ] es muy poco asibilada, tiende más bien a [I]. Auditivamente, se corrobora la sensación de muy poco asibilada.

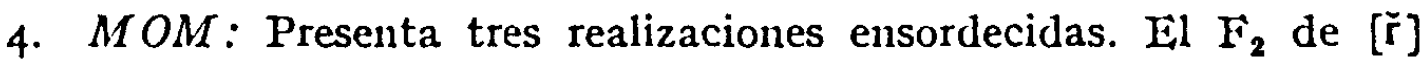
no aparece en seis casos; es armónico en 50 realizaciones e inarmónico en cuatro. Su fricación comienza a una frecuencia media de $2 \cdot 103,28 \mathrm{~Hz}$. Auditivamente es muy similar a la de OD. 
Los dos primeros informantes poseen una fricación más intensa y con un alcance de frecuencias más altas qne en los otros dos informantes, en los que la fricación es muy débil y de una gama de frecuencias bastante pequeña.

זo. Conclusiones generales.

De todo el material analizado, podemos obtener las siguientes conclusiones generales:

I. Duración. Salvo en el grupo [-š̌-], en todos los demás casos, [r] tiene mayor duración en sllaba tónica que en átona. Damos el cuadro resumen de todas las medias obtenidas en los apartados anteriores:

\begin{tabular}{|c|c|c|}
\hline & sll. tónica & sil. atona \\
\hline I. $[\check{r}-]$ & 12,8 & 8 \\
\hline 2. $[-\check{r}-]$ & 18,19 & 12,2 \\
\hline 3. $[-/ \bar{r}-]$ & 14 & 9,53 \\
\hline 4. $[-s \check{I}-]$ & 12,25 & 12,36 \\
\hline 5. $[-\mu \check{r}-]$ & 10,49 & 9.7 \\
\hline 6. $[-1 \check{r}-]$ & $I X, 2$ & 9 \\
\hline
\end{tabular}

Podemos observar cómo la duración mayor corresponde a [ř $]$ en posición intervocálica interior de palabra; le sigue la realización intervocálica, pero inicial de palabra, y después, la realización precedida de pausa. Lin [-š̃] la duración es practicamente la misma en cualquier posición acentual, y en los casos 5 y 6 existe muy poca variación, aunque en éstos la cantidad es menor que en el número 2. Los resultados que aqui muestran los casos $\mathrm{I}, 2$ y 3 dan pie para observar esas diferencias cuantitativas en los demás segmentos.

Gráficamente, representamos estas cantidades del siguiente modo en la fig. IO.I: 


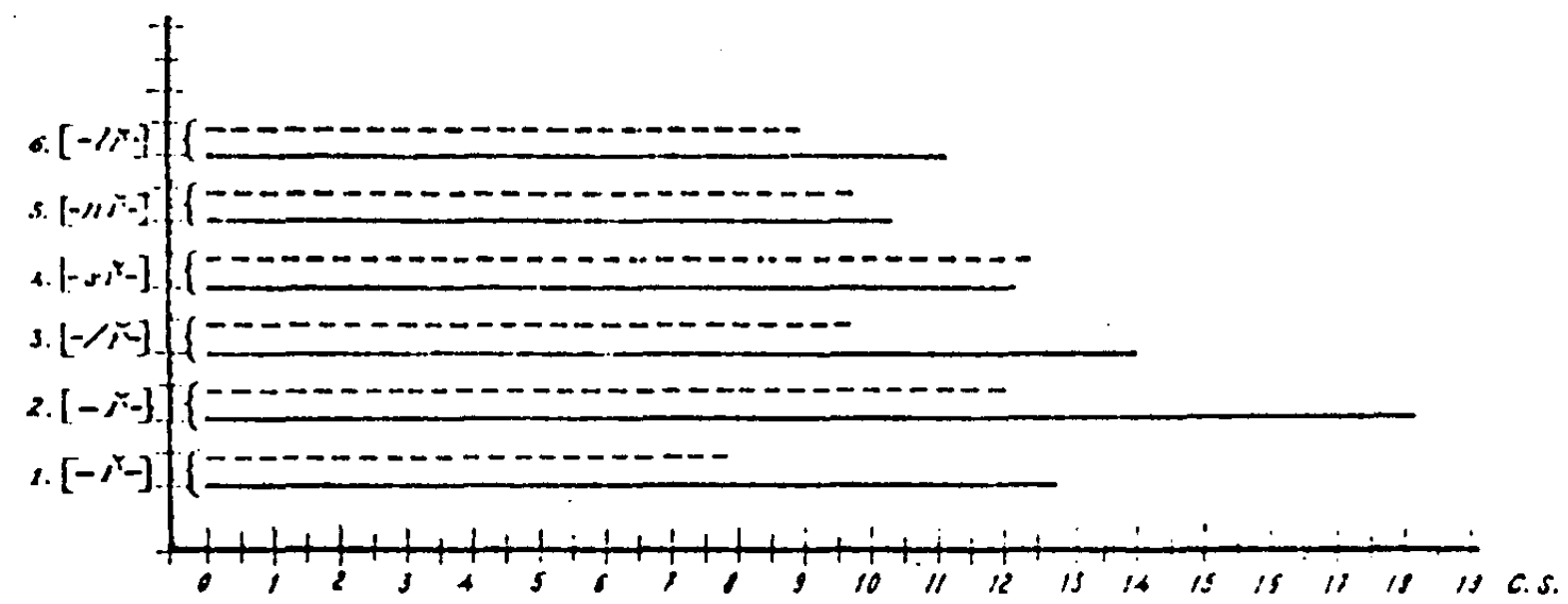

FIG. I0.1.

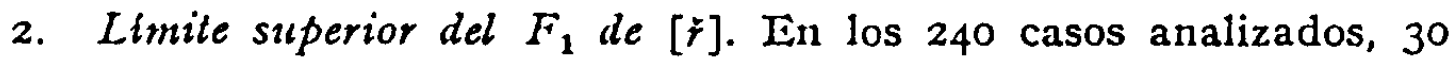
se han manifestado como totalmente ensordecidos. Los porcentajes de cnsordecimiento, en orden de mayor a menor frecuencia, son los siguientes: [-š́-]: $25 \% ;[\check{r}-]: 22,2 \% ;[-/ \check{r}-]: 18,3 \% ;[-\check{r}-]: 3,8 \% ;[-1 \grave{r}-]:$ $2,7 \%:[-1 \check{r}-]: 0 \%$. De nuevo $[-/ \check{r}-]$ está más próximo a $\left[\check{r}_{-}\right]$que a $[-\check{r}-]$.

En líneas generales, el ensordecimiento tiende a hacer $F_{2}$ inarmónico y a mostrar un comienzo más alto de la fricación.

E1 límite superior del primer formante de la consonante que nos ocupa varía entre los $457 \mathrm{~Hz}$ y los $557 \mathrm{~Hz}$. Los grupos de [consonan-

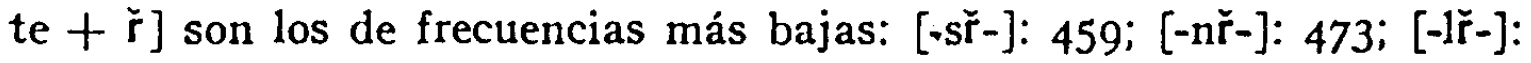
457. [ř -$]$ tiene la frecuencia más alta, $557 \mathrm{~Hz}$, seguido de $[-/ \check{r}-], 525 \mathrm{~Hz}$ $y$ de $[-\check{r}-], 509 \mathrm{~Hz}$.

3. Situación del $F_{2}$ de [r] . Con las vocales anteriores y central /i, e, a/ es más alto que con las vocales posteriores /o, u/. Además, para cada vocal ocupa una posición determinada:

Con /i/: cn ROM, 77r,5 Hz por clebajo clel $\mathrm{F}_{2}$ de la vocal en RGM, $957 \mathrm{~Hz}$ por debajo del $\mathrm{l}_{2}$, de la vocal en (U1), r.002 IIz por delsajo del $F_{2}$ de la vocal en MOM, x.068 $\mathrm{Hz}$ por debajo del $\mathrm{F}_{2}$ de la vocal

Con /c/: en ROM, $38 \mathrm{r} \quad \mathrm{Hz}$ por debajo del $\mathrm{F}_{2}$ de la vocal en RGM, $437 \mathrm{~Hz}$ por debajo del $\mathrm{F}_{2}$ de la vocal en $\mathrm{OD} . \quad 749 \mathrm{~Hz}$ por debajo del $\mathrm{F}_{2}$ de la vocal en MUM, $645 \mathrm{~Hz}$ por debajo del $\mathrm{F}$, de la vocal 
Con /a/: en ROM, $8 \mathrm{r} \quad \mathrm{Hz}$ por debajo del $\mathrm{F}_{\mathbf{z}}$ de la vocal eu RGM, $185,6 \mathrm{~Hz}$ por debajo del $\mathrm{F}_{2}$ de la vocal en OD. $\quad 38 \mathrm{~Hz}$ por debajo del $\mathrm{F}_{2}$ de la vocal en MOM, $616 \mathrm{~Hz}$ por debajo del $F_{1}$ de la vocal en ROM, 32I Hz por encima del li, de la vocal en RGM, 850 $\mathrm{Hz}$ por encina del $\mathrm{F}_{2}$ de la vocal eu $O D, \quad 8 \mathrm{r} H z$ por encima del $F_{8}$ de la vocal en ROM, al miswo nivel del $I_{3}$, de la vocal en $O D$, al mismo nivel del $F_{2}$ de la vocal

Con /o/: en ROM, i $76 \mathrm{~Hz}$ por encima del $\mathrm{r}_{2}$ de la vocal en RGM, $517 \mathrm{~Hz}$ por encima del $\mathrm{F}$, de la vocal en $O D, \quad 70 \quad H z$ por encima del $F_{2}$ de la vocal en MOM, $\quad 182 \mathrm{~Hz}$ por encina del $\mathrm{F}_{2}$ de la vocal en MOM, r67,8 $\mathrm{Hz}$ por debajo del $\mathrm{r}_{8}$ de la vocal

Con /u/: en ROM, $27 \mathrm{I} \quad \mathrm{Hz}$ por encima del $F_{2}$ de la vocal en RGM, $650 \mathrm{~Hz}$ por encima del $\mathrm{F}_{2}$ de la vocal en $O D, \quad$ I $90 \mathrm{~Hz}$ por encima del $\mathrm{F}_{2}$ de la vocal en MOM, $286 \mathrm{~Hz}$ por encima del $\mathrm{F}_{2}$ de la vocal

Cuando el $\mathrm{F}_{2}$ de $[\check{\mathrm{r}}$ ] forma sflaba con las vocales $/ \mathrm{i}, \mathrm{e} /$, se mantiene por debajo de los $F_{2}$ de las vocales mencionadas. Cuando forma silaba con $/ \mathrm{u} /$ aparece por encima. Con /a/, hay oscilaciones: al mismo nivel, por encima o por debajo del $\mathrm{F}_{2}$ de la vocal, sin que estas variaciones tengan correlación con la consonante anterior a [ř ].

El resumen gráfico de lo que acabamos de decir puede verse en la figura ro.2. En ella representamos los valores medios de los dos primeros formantes de cada vocal en cada informante y el segundo formante de $[\check{\mathbf{r}}]^{1}$.

4. Armonicidad/inarmonicidad del $F_{2}$ de [r̈]. De los 240 casos analizados, I22 han presentado un segundo formante de [r] plenamente armónico; en $\mathrm{I}_{4}$ realizaciones no se ha presentado el $\mathrm{F}_{2}, \mathrm{y}$ el resto estaban constitufdos por formantes inarmónicos.

Esta armonicidad o inarmonicidad repercute en:

a) la frecuencia a la que está situado este $\mathrm{F}_{2}$ : cuando es armónico, la frecuencia es más baja que cuando es inarmónico;

1 Los valores medios de $F_{1}$ y $F_{2}$ para cada vocal e iuformante son los siguientes: ROM: $[\mathrm{i}]=308-2.202 ;[\mathrm{e}]=377-\mathrm{r} .538,5 ;[\mathrm{a}]=624-\mathrm{r.897} ;[0]=380-\mathrm{o} .092 .5$; [u] = 295-924. RGM: $[\mathrm{i}]=304-3.116 ;[\mathrm{e}]=452-2.607 ;[\mathrm{a}]=925-\mathrm{x} .879 ;[0]=537-$ I.22x; $[\mathrm{u}]=3$ I8-1.028. OD: $[\mathrm{i}]=345^{-2.560 ;}[\mathrm{e}]=470-2.062 ;[\mathrm{a}]=87_{2-1.445 ;}$ $[0]=505-1.245 ;[\mathrm{u}]=34 \mathrm{I}-977$. MOM: $[\mathrm{i}]=344^{-2.5 I r ;}[\mathrm{e}]=45^{8-1.935 .5 i}[\mathrm{a}]=$ $=974-1.704 ;[0]=5$ I8-r.262; $[u]=405-926$. 

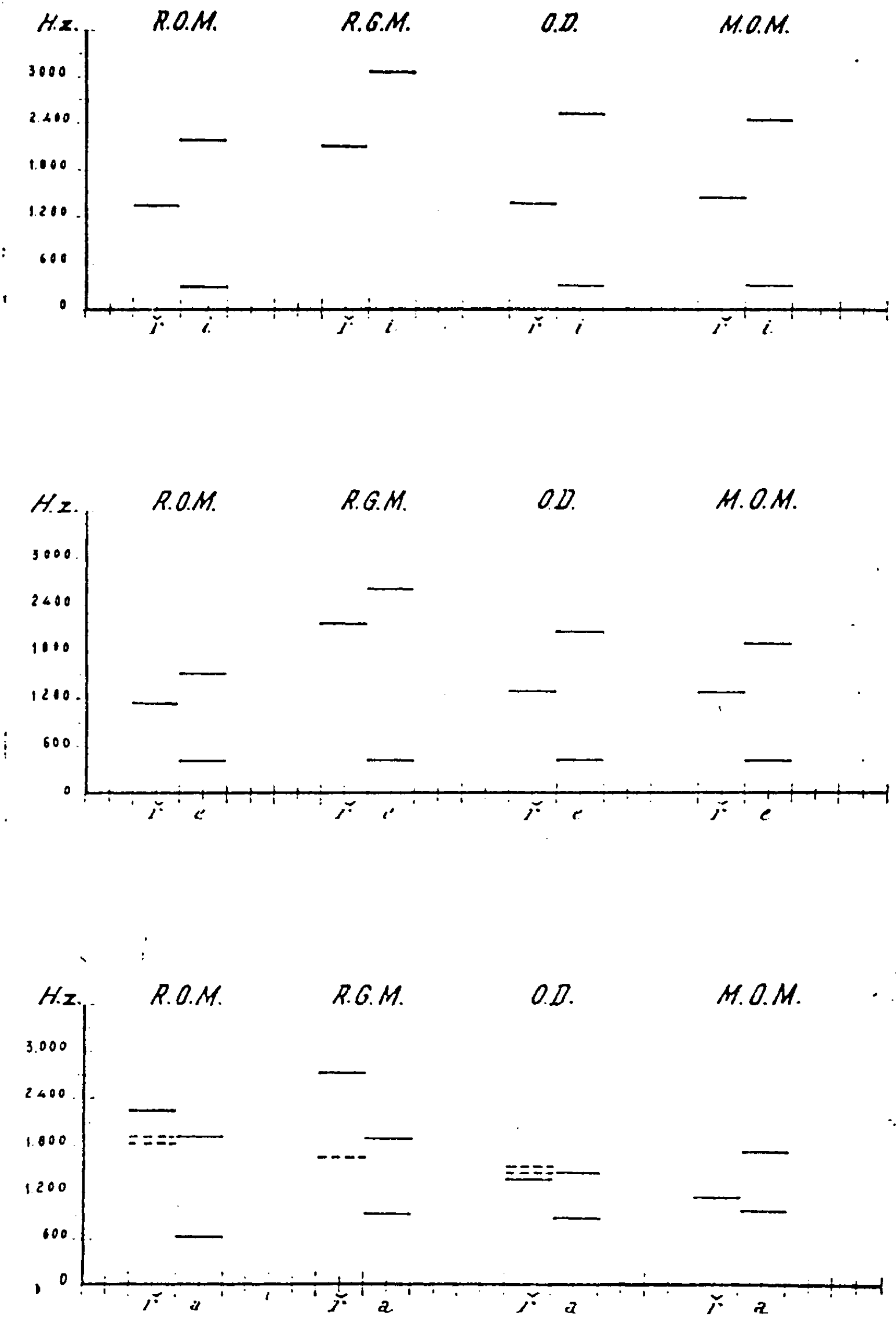

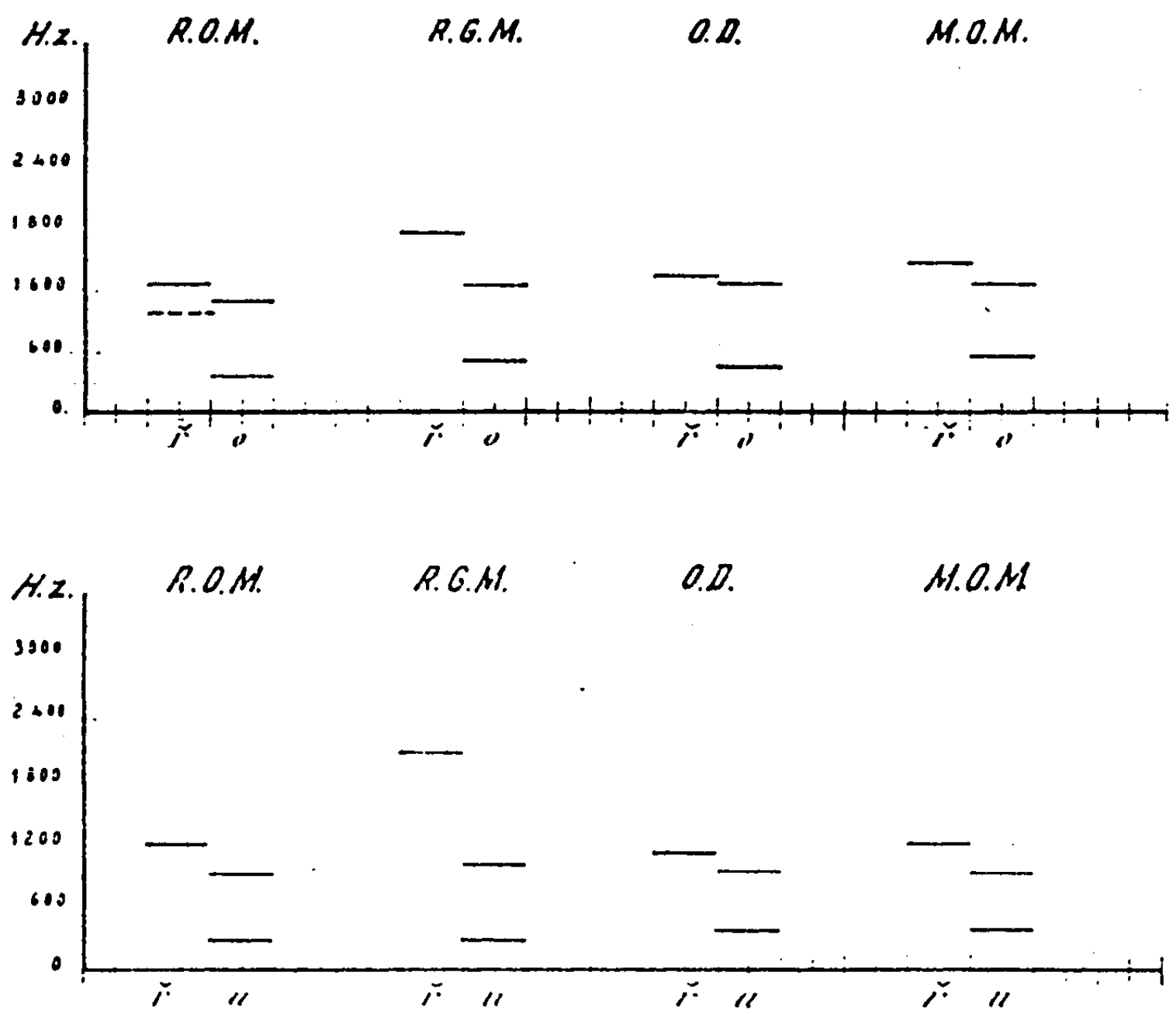

I'IG. 10.2 .

b) la frecuencia a la que comienza la fricación de [r] : cuando es armónico, la frecuencia también es más baja que cuando es inarmónico ${ }^{2}$.

En este aumento de frecuencia en el caso de la inarmonicidad nos parece ver un reflejo de tendencias hacia un mayor carácter sibilante.

5. El comienzo de la fricación de [ř ] se realiza por encima de su segundo formante, y es más alto con las vocales anteriores y central /i, e, a/ que con las posteriores $/ \mathrm{o}, \mathrm{u} /$. El descenso en contacto con estas últimas se debe al efecto de la labialización. Con las vocales anteriores y central, mantiene aproximadamente la misma altura, y lo mismo ocurre con las posteriores: ello nos indica que su lugar de articulación permanece estable cualquiera que sea el contorno vocálico en que se encuentra situado.

2 Cuando no aparece este segundo formante de [ř], el comienzo de la fricación se encuentra situada en un valor interwedio entre los valores que posec cuando es armónico e inarnónico. 
6. Las transiciones de los formantes de las vocales con las que forma sílaba [r̃ presentan los siguientes movimientos:

a) Con las vocales $/ \mathrm{i}, \mathrm{e} /$, son fuertemente negativas.

b) Con /a/ presentan bastantes variaciones: tanto pueden ser positivas, negativas $u$ horizontales, sin que veamos relación entre estos valores y la posición del $\mathrm{F}_{2}$ de $\left[\check{r}\right.$ ] respecto del $\mathrm{F}_{2}$ de [a]. (Estas transiciones $n$ son muy pronunciadas).

c) Con $/ 0 /$, los valores más generales son positivos, ya que, por regla general, el $\mathrm{F}_{2}$ de [ $\check{\mathrm{r}}$ ] está por encima de su segundo formante. Cuando éste está por debajo, la transición es negativa, y cuando está al mismo nivel, horizontal.

d) Como en el caso de $/ 0 /$, las transiciones de / $\mathrm{u} /$ varían con la posición del $\mathrm{F}_{2}$ de $[\tilde{r}]$ : cuando está por encima son positivas, negativas cuando está por debajo, y horizontales si se encuentra al mismo nivel.

De un modo general, podemos reflejar estas transiciones en el gráfico de la figura ro.3. Lin ella, los trazos continuos son los más numerosos.

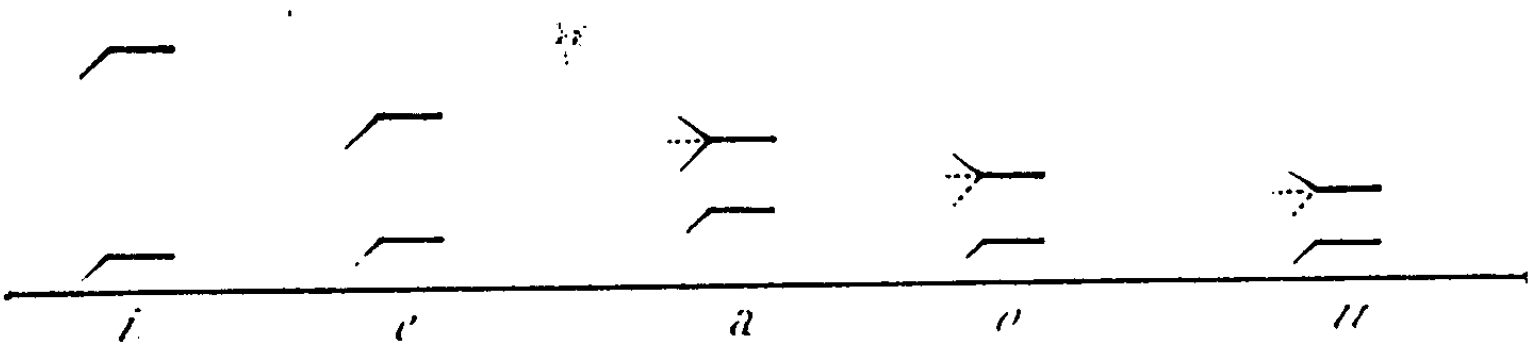

I'IG. 10.3.

7. Se distingue de [s] fundamentalmente: por la distinta altura en el comienzo de la fricación (mayor en [s]) y por la mayor altura frecuencial que alcanza el espectro de [s]; por la concentración de energía, que se produce a una frecuencia más baja en [ŕ ]; porque la anchura de la mayor concentración de energía es menor en [ $\tilde{\mathbf{r}}$ ] que en [s]; porque el carácter de la fricación es más estridente en [s]; por las transiciones del segundo formante, particularmente con $/ i, e, a /$; por la mayor intensidad de [s], y, sobre todo, en las [r̈] sonoras, por el primer formante.

8. Se distingue de [z]: por la frecuencia diferente del comienzo de la fricación: mayor en [Z]; el espectro fricativo de [z] alcanza valores más altos que el de [r] (aunque más bajos que [s]); la zona en la que 
se concentra la energía ocupa en [z] una posición intermedia entre [r] (más baja) y [s] (más alta); por las transiciones del $\mathrm{F}_{2}$, que son positivas. para [z] con todas las vocales, aumentando este carácter desde la zona anterior a la posterior.

\section{ANTONIO QUILIS \\ Ramón B. CarriL:}

Departamento de Fonética del C. S. I. C.

Universidad de Madrid. 